OPEN ACCESS

Edited by:

George Tsiamis,

University of Patras, Greece

Reviewed by:

Chao Wen,

Nanjing Agricultural University, China

Yuheng Luo,

Sichuan Agricultural University, China

Jianhua He,

Hunan Agricultural University, China

*Correspondence:

Jing Wang

wangjing@caas.cn

Specialty section

This article was submitted to

Systems Microbiology,

a section of the journa

Frontiers in Microbiology

Received: 30 November 2020 Accepted: 20 January 2021

Published: 22 February 2021

Citation:

Zhou J, Zhang H, Wu S, Qiu K,

Fu Y, Qi G and Wang J (2021)

Supplemental Xylooligosaccharide Modulates Intestinal Mucosal Barrier and Cecal Microbiota in Laying Hens Fed Oxidized Fish Oil.

Front. Microbiol. 12:635333. doi: 10.3389/fmicb.2021.635333

\section{Supplemental Xylooligosaccharide Modulates Intestinal Mucosal Barrier and Cecal Microbiota in Laying Hens Fed Oxidized Fish Oil}

\author{
Jian-min Zhou, Hai-jun Zhang, Shu-geng Wu, Kai Qiu, Yu Fu, Guang-hai Qi and \\ Jing Wang* \\ Laboratory of Quality and Safety Risk Assessment for Animal Products on Feed Hazards (Beijing) of the Ministry \\ of Agriculture and Rural Affairs, Feed Research Institute, Chinese Academy of Agricultural Sciences, Beijing, China
}

Our previous study indicated that dietary xylooligosaccharide (XOS) supplementation improved feed efficiency, ileal morphology, and nutrient digestibility in laying hens. The objective of this study was to evaluate the mitigative effects of XOS on intestinal mucosal barrier impairment and microbiota dysbiosis induced by oxidized fish oil (OFO) in laying hens. A total of 384 Hy-Line Brown layers at 50 weeks of age were randomly divided into four dietary treatments, including the diets supplemented with $20 \mathrm{~g} / \mathrm{kg}$ of fresh fish oil (FFO group) or $20 \mathrm{~g} / \mathrm{kg}$ of oxidized fish oil (OFO group), and the OFO diets with XOS addition at $200 \mathrm{mg} / \mathrm{kg}$ (OFO/XOS 200 group) or $400 \mathrm{mg} / \mathrm{kg}$ (OFO/XOS 400 group). Each treatment had eight replicates with 12 birds each. The OFO treatment decreased $(P<0.05)$ the production performance of birds from 7 to 12 weeks of the experiment, reduced $(P<0.05)$ ileal mucosal secretory immunoglobulin A (slgA) content, and increased $(P<0.05)$ serum endotoxin concentration, as well as downregulated $(P<0.05)$ mRNA expression of claudin-1 (CLDN1) and claudin-5 (CLDN5) in the ileal mucosa at the end of the experiment. Dietary XOS addition $(400 \mathrm{mg} / \mathrm{kg})$ recovered $(P<0.05)$ these changes and further improved $(P<0.05)$ ileal villus height $(\mathrm{VH})$ and the villus height-to-crypt depth ratio (VCR). In addition, OFO treatment altered cecal microbial composition of layers, and these alterations were probably involved in OFO-induced ileal mucosal impairment as causes or consequences. Supplemental XOS remodeled cecal microbiota of layers fed the OFO diet, characterized by an elevation in microbial richness and changes in microbial composition, including increases in Firmicutes, Ruminococcaceae, Verrucomicrobia (Akkermansia), Paraprevotella, Prevotella_9, and Oscillospira, along with a decrease in Erysipelatoclostridium. The increased abundance of Verrucomicrobia (Akkermansia) had positive correlations with the improved ileal $\mathrm{VH}$ and ileal mucosal expression of CLDN1. The abundance of Erysipelatoclostridium decreased by XOS addition was negatively associated with ileal $\mathrm{VH}, \mathrm{VCR}$, ileal mucosal slgA content, and the relative 
expression of zonula occludens-2, CLDN1, and CLDN5. Collectively, supplemental XOS alleviated OFO-induced intestinal mucosal barrier dysfunction and performance impairment in laying hens, which could be at least partially attributed to the modulation of gut microbiota.

Keywords: oxidized fish oil, laying hen, intestinal mucosal barrier, gut microbiota, XOS

\section{INTRODUCTION}

The intestinal mucosa is a dynamic interface and mainly consists of epithelial cells linked by tight junctions, separating hostile external elements from internal milieu (Sánchez de Medina et al., 2014). Functionally, the intestinal mucosal barrier not only guarantees nutrient absorption but also ensures adequate containment of detrimental luminal contents through the turnover of mucus, production of secretory immunoglobulin A (sIgA), and maintenance of tight junctions (Derrien et al., 2017; Xie et al., 2019a). However, some harmful ingredients in laying hen diets can damage the intestinal mucosal barrier and further elicit translocation of pathogens, progression of inflammation, and dysfunction of nutrient absorption, resulting in poor performance and economic efficiency (Xing et al., 2019; Miao et al., 2020). Fish oil rich in $n$-3 polyunsaturated fatty acids (PUFA) has been widely added to layer diets to produce n-3 PUFA-enriched eggs (Dong et al., 2018; Feng et al., 2019). Nevertheless, high $n$-3 PUFA content in fish oil also means that it is easily oxidized, generating primary and secondary lipid peroxidation products. These toxicants such as lipid hydroperoxides are toxic substances that can damage the intestinal epithelial cells by reducing membrane integrity and disturbing membrane permeability of birds (Dibner et al., 1996; Dasilva et al., 2017). Tan et al. (2019) demonstrated that supplementation of broiler diets with $4 \%$ oxidized fish oil (OFO) decreased the expression of tight junction proteins and upregulated the expression of pro-inflammatory cytokine $I L-22$ in the intestinal mucosa. Previous publications have shown that the use of antioxidants such as ascorbic acid and polyphenols are effective in preventing lipid oxidation during gastrointestinal digestion (Lapidot et al., 2005; Maestre et al., 2013). However, there is limited information concerning the repair of intestinal mucosal barrier induced by oxidized lipids.

Xylooligosaccharide (XOS) is a sugar oligomer of two to seven xylose units linked by $\beta$-1,4-glycosidic bonds (Jordan and Kurt, 2010). Although chickens lack enzymes required to degrade the glycoside links between xylose units, XOS is available for fermentation in the cecum by xylanolytic bacteria (Pourabedin and Zhao, 2015). Selective fermentation of XOS in turn shifted the gut microbiota toward a relative increase in probiotics and a decrease in pathogenic bacteria, thus contributing to the improvements in intestinal health and immune function in poultry (De Maesschalck et al., 2015; Ding et al., 2018; Lin et al., 2018). It was reported that XOS addition enhanced

Abbreviations: XOS, xylooligosaccharide; OFO, oxidized fish oil; FFO, fresh fish oil; SIgA, secretory immunoglobulin A; VH, villus height; CD, crypt depth; VCR, the villus height to crypt depth ratio; ZO2, zonula occludens-2; CLDN1, claudin-1; CLDN5, claudin-5; PUFA, polyunsaturated fatty acids. the intestinal barrier function by upregulating the expression of tight junction protein occludin in rats (Christensen et al., 2014). Similarly, dietary supplementation of prebiotics and synbiotics (both contained $150 \mathrm{mg} / \mathrm{kg}$ XOS) improved small intestinal morphology and sIgA content in broilers (Min et al., 2016). Besides, XOS was also demonstrated to mitigate highfat diet-induced intestinal inflammation and barrier dysfunction by modulating gut microbial composition (Fei et al., 2020). Accordingly, addition of XOS may exert active roles in protecting intestinal function in chickens. The current study was aimed to investigate the positive effects of XOS addition on gut microbiota and intestinal mucosal barrier in laying hens fed OFO.

Gut microbiota composition is closely linked to the intestinal barrier function, including the modulations of epithelial shield, mucus layer, and mucosal immunity (Alam and Neish, 2018). Although our previous study has identified that XOS addition can improve feed efficiency and ileal morphology, and modulate cecal microbial composition (Zhou et al., 2020), it is still unknown whether XOS addition can protect intestinal mucosal barrier from damage through regulating gut microbiota dysbiosis. In keeping with this, the objective of this study was to assess the alteration of gut microbiota to explain the possible mechanism through which XOS alleviates OFO-induced impairment of intestinal mucosal barrier.

\section{MATERIALS AND METHODS}

\section{Ethics Statement}

All experimental protocols were approved by the Animal Care and Use Committee of the Feed Research Institute of the Chinese Academy of Agricultural Sciences (ACE-CAAS-20180915), and the methods were carried out in accordance with the relevant guidelines and regulations.

\section{Fish Oil Preparation}

Fresh fish oil (FFO) without any antioxidant was purchased from the Foshan Fish Oil Production Company (Guangdong, China) and stored in a refrigerator at $-20^{\circ} \mathrm{C}$ prior to their addition to feed. The oxidized fish oil was prepared by heating the fresh fish oil at $90^{\circ} \mathrm{C}$ with vigorous aeration for $72 \mathrm{~h}$. Peroxide values of fresh and oxidized fish oil analyzed according to the ISO method ISO 3960 (International Organization for Standarization [ISO], 2001) were 3.20 and 22.25 $\mathrm{meq} / \mathrm{kg}$, respectively.

\section{Birds and Experimental Design}

A total of 384 Hy-Line Brown layers aged 50 weeks were randomly allocated into four groups, with eight replicates (12 
birds in four adjacent cages as a replicate). Initial body weight and egg production were similar across all the replicates. The treatment groups were as follows: FFO group, birds were fed diet with $20 \mathrm{~g} / \mathrm{kg}$ fresh fish oil; OFO group, birds were fed diet with $20 \mathrm{~g} / \mathrm{kg}$ of oxidized fish oil; OFO/XOS 200 group, birds were fed diet with $20 \mathrm{~g} / \mathrm{kg}$ of oxidized fish oil, and $200 \mathrm{mg} / \mathrm{kg}$ of XOS; and $\mathrm{OFO} / \mathrm{XOS}_{400}$ group, birds were fed diet with $20 \mathrm{~g} / \mathrm{kg}$ of oxidized fish oil and $400 \mathrm{mg} / \mathrm{kg}$ of XOS. Laying hens for the trial were allocated to three-tier battery cages of three laying hens each (cage size: $40 \mathrm{~cm} \times 40 \mathrm{~cm} \times 35 \mathrm{~cm}$ ) and exposed to $16 \mathrm{~h}$ of light/day with an intensity of $20 \mathrm{~lx}$. Temperature of layer house was affected by seasonal change, which was pretty higher in the first 6 weeks (between 27 and $32^{\circ} \mathrm{C}$ ) than that in the last 6 weeks (between 24 and $28^{\circ} \mathrm{C}$ ). Diets and water were offered ad libitum in mash form and by nipple drinkers, respectively. All hens remained in good health during the feeding period. There were no culled birds, and medical intervention was not applied to any bird. The experimental diet (Table 1) was formulated according to the National Research and Council (1994). The XOS (XOS95P) added in the diets was purchased from a commercial supplier (Jinan Longlive Biology Co., Ltd., Shandong, China). It was extracted from corncob and contained 95\% XOS with a degree of polymerization of from 2 to 7 and $5 \%$ xylose on the dry matter basis. The XOS was added in the diets at the expense of corn.

\section{Performance Measurement}

Mortality was recorded as it occurred. Daily egg number, total egg weight, and biweekly feed consumption were recorded and calculated as hen-day egg production (EP), average egg weight (AEW), average daily feed intake (ADFI), feed conversion ratio (FCR) on a biweekly basis. EP, AEW, ADFI, and FCR were calculated for weeks 1-6, 7-12, and 1-12.

\section{Sample Collection}

At the end of the feeding trial, one bird per replicate was randomly selected for sample collection. Blood samples were collected from wing vein, serum was isolated by centrifugation at $3,000 \times \mathrm{g}$ for $10 \mathrm{~min}$ at $4^{\circ} \mathrm{C}$ and stored at $-20^{\circ} \mathrm{C}$ for endotoxin analysis. After blood collection, these birds were slaughtered rapidly. Segments (approximately $2 \mathrm{~cm}$ in length) of the middle portion of the ileum (about $5 \mathrm{~cm}$ from Meckel's diverticulum) were collected, washed with PBS, and fixed in $10 \%$ neutral-buffered formalin for histology. The remainder of ileum was removed, opened longitudinally, and gently rinsed with PBS. The mucosa was scraped aseptically by sterile glass slides, frozen as aliquots in liquid nitrogen, and stored at $-80^{\circ} \mathrm{C}$ for $\operatorname{sIgA}$ determination and the quantification of gene expression. Additionally, cecal contents (approximately $1 \mathrm{~g}$ ) were collected in sterile containers, and then frozen by immersion in liquid nitrogen, and stored at $-80^{\circ} \mathrm{C}$ for intestinal microbiota analysis.

\section{Ileal Morphology Analysis}

Samples were washed, dehydrated, clarified, and embedded in paraffin. Serial sections were cut at $5 \mu \mathrm{m}$ thickness, placed on glass slides, deparaffinized in xylene, rehydrated, stained with hematoxylin and eosin, fixed with neutral balsam, and examined by light microscopy (BX51, Olympus Co., Tokyo, Japan). All reagents used were of analytical grade (Sinopharm Chemical Reagent Co., Ltd., Beijing, China). The morphometric indices evaluated were villus height $(\mathrm{VH}$; from the tip of the villus to the villus-crypt junction), crypt depth (CD; from the base up to the crypt-villus transition region), and the villus height-to-crypt depth ratio (VCR) (Forte et al., 2016). The number of goblet cells (GCN) was counted on 100 columnar cells of villus mucosa at $400 \times$ magnification.

\section{Content of SIgA in the lleal Mucosa}

Ileal mucosa was homogenized in 10 volumes of ice-cold saline and centrifuged at $20,000 \times \mathrm{g}$ for $10 \mathrm{~min}$ at $4^{\circ} \mathrm{C}$. The supernatant was collected for sIgA determination using an assay kit (Jiancheng Bioengineering Institute, Nanjing, China). All the procedures were carried out according to the instructions of the manufacturer. The protein concentration was detected by the Coomassie Brilliant Blue method, using an assay kit (Jiancheng Bioengineering Institute, Nanjing, China), and the sIgA content was expressed as $\mathrm{ng} / \mathrm{g}$ protein.

TABLE 1 | Composition and nutrient levels of experimental diet (as-fed basis).

\begin{tabular}{lc}
\hline Ingredient & Content (\%) $^{\mathbf{d}}$ \\
\hline Corn & 62.78 \\
Soybean meal (44\% crude protein) & 24.00 \\
Fresh or oxidized fish oil $^{\mathrm{a}}$ & 2.00 \\
Salt & 0.30 \\
Dicalcium phosphate & 1.00 \\
Calcium carbonate & 9.34 \\
DL-Methionine & 0.12 \\
Choline chloride (50\%) & 0.10 \\
Premix & 0.33 \\
Phytase & 0.03 \\
XOS95P & $\pm{ }^{\mathrm{e}}$ \\
Nutrient level & \\
Metabolizable energy (MJ/kg) & 11.64 \\
Crude protein & $16.5(16.34)$ \\
Non-phytate phosphorus & 0.33 \\
Calcium & 3.48 \\
Lysine & 0.79 \\
Methionine & 0.37 \\
Methionine + cysteine & 0.65 \\
\hline a & 0.60 \\
\hline
\end{tabular}

aperoxide values of fresh and oxidized fish oil were 3.20 and $22.25 \mathrm{meq} / \mathrm{kg}$, respectively.

${ }^{b}$ Premix supplied per kilogram of diet: vitamin A, 12,500 U; vitamin $D_{3}, 4,125 \mathrm{U}$; vitamin E, $15 \mathrm{U}$; vitamin $\mathrm{K}_{3}, 2 \mathrm{mg}$; thiamine, $1 \mathrm{mg}$; riboflavin, $8.5 \mathrm{mg}$; pyridoxine, $8 \mathrm{mg}$; vitamin $B_{12}, 0.04 \mathrm{mg}$; biotin, $0.1 \mathrm{mg}$; folic acid, $1.25 \mathrm{mg}$; Ca-pantothenate, 50 mg; niacin, 32.5 mg; Cu, 8 mg; Zn, 65 mg; Fe, 60 mg; Mn, 65 mg; Se, 0.3 mg; I, $1 \mathrm{mg}$.

${ }^{c}$ XOS95P, a mixture of 95\% xylooligosaccharide (XOS) with the degree of polymerization 2-7 and 5\% xylose on the dry matter basis.

${ }^{d}$ The value in parenthesis indicates analyzed value. Others are calculated values.

eXOS was supplemented at 200 and $400 \mathrm{mg} / \mathrm{kg}$ at the expense of corn. 


\section{Concentration of Endotoxin in Serum}

Birds in the OFO/XOS 400 group exhibited the greater ileal morphology and ileal mucosal sIgA content than those in the $\mathrm{OFO} / \mathrm{XOS}_{200}$ group. Therefore, further determinations including serum endotoxin concentration, relative mRNA expression of tight junction proteins, and cecal microbial diversity and composition were only conducted among the $\mathrm{FFO}, \mathrm{OFO}$, and $\mathrm{OFO} / \mathrm{XOS}_{400}$ groups to reveal the possible mechanism of the alleviation of XOS on intestinal mucosal impairment induced by OFO. Serum endotoxin concentration was measured using a chromogenic substrate assay kit (Jiancheng Bioengineering Institute, Nanjing, China). Briefly, $0.1 \mathrm{ml}$ of serum was incubated with $0.1 \mathrm{ml}$ of Limulus amebocyte lysate at $37^{\circ} \mathrm{C}$ for $45 \mathrm{~min}$. After several subsequent reactions, the samples were read spectrophotometrically at $545 \mathrm{~nm}$. The endotoxin level was calculated based on a standard curve of endotoxin concentration.

\section{Quantitative Real-Time PCR Analysis}

Total RNA of the ileal mucosa was extracted using Trizol reagent (Tiangen Biotech Co., Ltd., Beijing, China) according to the manufacturer's instructions. RNA concentration was determined using a NanoDrop 2000 spectrophotometer (Thermo Fisher Scientific, Waltham, MA), and the integrity was evaluated using agarose-ethidium bromide electrophoresis. Reverse transcription (RT) reactions were immediately performed using the FastQuant RT Kit (Tiangen Biotech Co., Ltd., Beijing, China). Real-time quantitative PCR was conducted in duplicate in the Bio-Rad C1000 thermal cycler (CFX-96 real-time PCR detection systems; Bio-Rad). PCR programs for all genes were as follows: $15 \mathrm{~min}$ at $95^{\circ} \mathrm{C}, 40$ cycles of $95^{\circ} \mathrm{C}$ for $10 \mathrm{~s}, 60^{\circ} \mathrm{C}$ for $30 \mathrm{~s}$. The relative gene expression levels were calculated using the $2^{-\Delta \Delta C t}$ method (Livak and Schmittgen, 2001), and avian $\beta$-actin was used as reference gene. The primer sequences for the target genes [zonula occludens-2 (ZO2), claudin-1 (CLDN1), claudin-5 (CLDN5)] and $\beta$-actin are listed in Table 2.

\section{DNA Extraction, 16S rRNA Amplification, and High-Throughput Sequencing}

Total bacterial genomic DNA was extracted from cecal content samples using the E.Z.N.A Soil DNA Kit (Omega Bio-tek,

TABLE 2 | Primers used for quantitative real-time PCR.

\begin{tabular}{|c|c|c|c|}
\hline Gene $^{a}$ & Primer sequences & Accession number & Length (bp) \\
\hline \multirow[t]{2}{*}{$\mathrm{ZO2}$} & F:CGGCAGCTATCAGACCACTC & NM_204918 & 87 \\
\hline & R:CACAGACCAGCAAGCCTACAG & & \\
\hline \multirow[t]{2}{*}{ CLDN1 } & F:CTGATTGCTTCCAACCAG & NM_001013611 & 140 \\
\hline & R:CAGGTCAAACAGAGGTACAAG & & \\
\hline \multirow[t]{2}{*}{ CLDN5 } & F:CATCACTTCTCCTTCGTCAGC & NM_204201 & 111 \\
\hline & R:GCACAAAGATCTCCCAGGTC & & \\
\hline \multirow[t]{2}{*}{$\beta$-actin } & F:ATGATATTGCTGCGCTCGTT & NM_205518.1 & 145 \\
\hline & R:TCTTCTGGCCCATACCAACC & & \\
\hline
\end{tabular}

aZO2, zona occludens-2; CLDN1, claudin-1; CLDN5, claudin-5.
Norcross, GA, United States) according to manufacturer's instructions. The quality of DNA samples was checked with gel electrophoresis. Bacterial 16S rDNA sequences spanning the hypervariable regions $\mathrm{v} 3-\mathrm{v} 4$ were amplified using primers: 338F (5'-ACT CCT ACG GGA GGC AGC A-3') and 806R (5'GGA CTA CHV GGG TWT CTA AT- $3^{\prime}$ ). The PCR reaction conditions were $2 \mathrm{~min}$ of denaturation at $95^{\circ} \mathrm{C}$, followed by 25 cycles of $30 \mathrm{~s}$ at $95^{\circ} \mathrm{C}$ (denaturation), annealing at $55^{\circ} \mathrm{C}$ for $30 \mathrm{~s}$, and extension at $72^{\circ} \mathrm{C}$ for $30 \mathrm{~s}$, with a final extension of $5 \mathrm{~min}$ at $72^{\circ} \mathrm{C}$. Amplicons were extracted from $2 \%$ agarose gels and purified using the AxyPrep DNA Gel Extraction Kit (Axygen Biosciences, Union City, CA, United States) according to the manufacturer's instructions. Purified amplicons were qualified and sequenced using MiSeq platform with MiSeq Reagent Kit v3 at Shanghai Personal Biotechnology Co., Ltd. (Shanghai, China). The raw reads were deposited into the NCBI Sequence Read Archive (SRA) database (Accession Number: PRJNA673331). The raw pair-end sequences from the original DNA fragments were demultiplexed and quality-filtered using the Quantitative Insights Into Microbial Ecology (QIIME, version 1.17). Only sequences that overlap by more than $10 \mathrm{bp}$ were assembled according to their overlap sequence. Operational taxonomic units (OTUs) were clustered with 97\% sequence identity using UPARSE (version 7.1), and the chimera sequences were identified and removed to obtain effective tags using UCHIME. Shannon and Simpson diversity indices, and Ace and Chao richness estimators were included in the alpha diversity analysis by using the MOTHUR v1.31.2. Principal coordinate analysis (PCoA) and partial least squares discriminant analysis (PLS-DA) based on unweighted UniFrac distance were conducted to compare the bacterial community structures across all samples and to establish beta diversity. The significance of differentiation of microbial structure among groups was statistically tested by analysis of similarity (ANOSIM). Statistical tests for differentially abundant taxa were performed using the linear discriminant analysis (LDA) effect size (LEfSe) method with an alpha value of 0.05 for the Kruskal-Wallis test among classes, and the threshold for the $\log _{10} \mathrm{LDA}$ score was set as 2.0. Metagenome functional content from 16S rDNA was predicted using PICRUSt (Langille et al., 2013), based on the Clusters of Orthologous Groups (COG) and Kyoto Encyclopedia Genes and Genomes (KEGG) databases. Pearson correlation analysis was performed for the correlations between phylotypes and phenotypes.

\section{Statistical Analysis}

Differences in performance, ileal morphology, ileal mucosal sIgA, serum endotoxin, relative expression of tight junction proteins, and alpha diversity indices of cecal microbiota were analyzed using one-way ANOVA followed by Duncan's multiple range test (SPSS, version 19.0, Chicago, IL). Wilcoxon rank-sum test was employed to explore the differences in cecal microbiota in terms of the relative abundances of species between groups FFO and OFO, and between groups $\mathrm{OFO}$ and $\mathrm{OFO} / \mathrm{XOS}_{400}$. Significance was set at $P<0.05$, and $0.05<P<0.10$ was considered as a trend toward significance. Data are expressed as the means and pooled SEM. 


\section{RESULTS}

\section{Performance}

No birds died during entire experimental period. Birds in the OFO group presented a lower $(P<0.05)$ egg production from weeks 7 to 12 of the experiment, which was concomitant with poor $(P<0.10)$ FCR from weeks 7 to 12 and 1 to 12 than those in the FFO group (Table 3). However, there were no significant differences in EP and FCR among the FFO, $\mathrm{OFO} / \mathrm{XOS}_{200}$, and $\mathrm{OFO} / \mathrm{XOS}_{400}$ groups from weeks 7 to 12 or 1 to $12(P>0.05)$. Affected by the temperature of layer house, egg production of all birds in the first 6 weeks was lower than that in the second 6 weeks.

\section{lleal Morphology}

The ileal morphology of laying hens fed the experimental diets is shown in Table 4 and Supplementary Figure S1. No significant differences were detected in ileal VH, CD, the VCR, or GCN between FFO and OFO groups $(P>0.05)$. However, hens in the $\mathrm{OFO} / \mathrm{XOS}_{400}$ group showed higher $\mathrm{VH}, \mathrm{VCR}$, and GCN in the ileum compared with those in the other groups $(P<0.05)$.

\section{Concentrations of SIgA in the lleal Mucosa and the Endotoxin in Serum}

The content of sIgA in the ileal mucosa of laying hens at week 12 is shown in Figure 1A. There was a significant decrease $(P<0.05)$ in ileal mucosal sIgA content of hens in the OFO and

TABLE 3 | Effect of dietary xylooligosaccharide supplementation on performance of laying hens fed an oxidized fish oil diet ${ }^{1}$.

\begin{tabular}{|c|c|c|c|c|c|c|}
\hline \multirow[t]{2}{*}{ Item $^{2}$} & \multicolumn{4}{|c|}{ Experimental treatment ${ }^{3}$} & \multirow[t]{2}{*}{ SEM } & \multirow[t]{2}{*}{$P$-value } \\
\hline & FFO & OFO & $\mathrm{OFO} \mathrm{XOS}_{200}$ & $\mathrm{OFO} \mathrm{XOS}_{400}$ & & \\
\hline \multicolumn{7}{|c|}{ Weeks 1-6 } \\
\hline EP (\%) & 88.00 & 87.67 & 87.17 & 86.14 & 0.75 & 0.849 \\
\hline AEW (g) & 63.25 & 63.10 & 63.24 & 63.81 & 0.36 & 0.915 \\
\hline ADFI (g) & 114.93 & 114.17 & 112.26 & 113.59 & 0.72 & 0.625 \\
\hline FCR & 2.01 & 2.01 & 1.98 & 2.00 & 0.02 & 0.932 \\
\hline \multicolumn{7}{|c|}{ Weeks 7-12 } \\
\hline EP (\%) & $93.78^{a}$ & $88.34^{b}$ & $91.08^{\mathrm{ab}}$ & $90.58^{a b}$ & 0.61 & 0.011 \\
\hline AEW (g) & 65.60 & 65.18 & 65.45 & 65.76 & 0.34 & 0.949 \\
\hline ADFI (g) & 120.34 & 121.15 & 119.62 & 119.20 & 0.45 & 0.445 \\
\hline FCR & 1.99 & 2.11 & 2.02 & 2.04 & 0.02 & 0.051 \\
\hline \multicolumn{7}{|c|}{ Weeks 1-12 } \\
\hline EP (\%) & 90.80 & 87.73 & 89.03 & 88.08 & 0.51 & 0.139 \\
\hline AEW (g) & 64.42 & 64.14 & 64.34 & 64.78 & 0.34 & 0.935 \\
\hline ADFI (g) & 117.63 & 117.96 & 115.94 & 116.40 & 0.51 & 0.553 \\
\hline FCR & 1.96 & 2.05 & 2.00 & 2.03 & 0.01 & 0.066 \\
\hline
\end{tabular}

${ }^{1} n=8$ replicates per treatment.

${ }^{2} E P$, egg production; $A E W$, average egg weight; $A D F I$, average daily feed intake; $F C R$, feed conversion ratio (feed/egg; $g / g$ ).

${ }^{3} \mathrm{FFO}$, fresh fish oil diet; OFO, oxidized fish oil diet; OFO/XOS 200 , oxidized fish oil diet $+200 \mathrm{mg} / \mathrm{kg}$ xylooligosaccharide; OFO/XOS 400 , oxidized fish oil diet $+400 \mathrm{mg} / \mathrm{kg}$ xylooligosaccharide.

$a, b$ Within a row, means with no common small letters $(a-c)$ differ significantly $(P<0.05)$.
TABLE 4 | Effect of dietary xylooligosaccharide supplementation on ileal morphology of laying hens fed an oxidized fish oil diet ${ }^{1}$.

\begin{tabular}{|c|c|c|c|c|c|c|}
\hline \multirow[t]{2}{*}{ Item $^{2}$} & \multicolumn{4}{|c|}{ Experimental treatment ${ }^{3}$} & \multirow[t]{2}{*}{ SEM } & \multirow[t]{2}{*}{$\boldsymbol{P}$-value } \\
\hline & FFO & OFO & OFO/XOS $_{200}$ & OFO/XOS $_{400}$ & & \\
\hline $\mathrm{VH}(\mu \mathrm{m})$ & $611.85^{\mathrm{b}}$ & $594.94^{b}$ & $613.40^{b}$ & $749.70^{a}$ & 19.05 & 0.007 \\
\hline $\mathrm{CD}(\mu \mathrm{m})$ & 159.28 & 164.75 & 161.19 & 165.09 & 4.32 & 0.691 \\
\hline VCR & $3.99^{b}$ & $3.64^{b}$ & $3.82^{b}$ & $4.59^{\mathrm{a}}$ & 0.10 & 0.002 \\
\hline GCN & $21.13^{b}$ & $19.33^{b}$ & $20.23^{b}$ & $24.91^{\mathrm{a}}$ & 0.55 & $<0.001$ \\
\hline
\end{tabular}

${ }^{1} n=8$ replicates per treatment.

${ }^{2} \mathrm{VH}$, villus height; $\mathrm{CD}$, crypt depth; VCR, the villus height to crypt depth ratio; GCN, goblet cells number.

${ }^{3} \mathrm{FFO}$, fresh fish oil diet; OFO, oxidized fish oil diet; OFO/XOS 200 , oxidized fish oil diet $+200 \mathrm{mg} / \mathrm{kg}$ xylooligosaccharide; OFO/XOS 400 , oxidized fish oil diet $+400 \mathrm{mg} / \mathrm{kg}$ xylooligosaccharide.

a,b Within a row, means with no common small letter differ significantly $(P<0.05)$.

$\mathrm{OFO} / \mathrm{XOS}_{200}$ groups, compared with the FFO group; however, it was higher $(P<0.05)$ in the $\mathrm{OFO} / \mathrm{XOS}_{400}$ group than in the OFO group.

The increased $(P<0.05)$ serum endotoxin concentration induced by the oxidized fish oil diet was diminished by $400 \mathrm{mg} / \mathrm{kg}$ of XOS supplementation, reflected by no significant difference between FFO and OFO/XOS 400 groups $(P>0.05)$ (Figure 1B).

\section{Relative mRNA Expression of Tight Junction Proteins}

There were decreases $(P<0.05)$ in the relative expression of ileal mucosal CLDN1 and CLDN5 of the layers in the OFO group, compared with the FFO group (Figure 1C); however, they were both increased $(P<0.05)$ in the layers of the OFO/XOS 400 group when compared with those in the OFO group.

\section{Diversity of Gut Microbiota}

Bacterial alpha diversity in cecal microbiota was estimated using Shannon, Simpson, Ace, and Chao indices of diversity and richness (Figure 2). No significant differences in Shannon or Simpson indices were found among the 3 groups $(P>0.05)$. Supplemental XOS $(400 \mathrm{mg} / \mathrm{kg})$ increased Ace $(P=0.028)$ and Chao $(P=0.084)$ indices of the cecal microbiota in laying hens fed oxidized fish oil. PCoA based on unweighted UniFrac distance revealed a separation of microbial communities among the 3 groups (PCo1, 15.88\%; PCo2, 13.73\%) (Figure 3A). PLS-DA plot also defined groups where samples from different groups occupied distinct positions (COMP1, 11.29\%; COMP2, 6.44\%) (Figure 3B). Results were supported by statistics obtained from ANOSIM analysis (Supplementary Table S1).

\section{Composition of Gut Microbiota}

Taxonomic compositions of the cecal microbiota were analyzed at the different levels using the RDA classifier. The dominant phyla across all the groups were Bacteroidetes and Firmicutes, which together contributed greater than $88 \%$ of the whole phyla (Figure 4A). A higher abundance of Bacteroidetes with a lower abundance of Firmicutes were observed in layers fed OFO compared to those in the FFO group. However, 
A

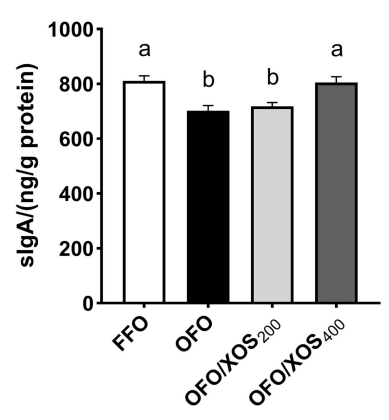

B

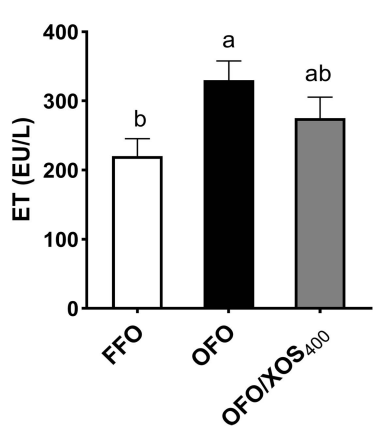

C

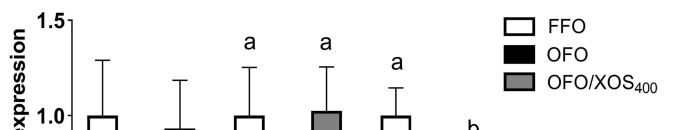

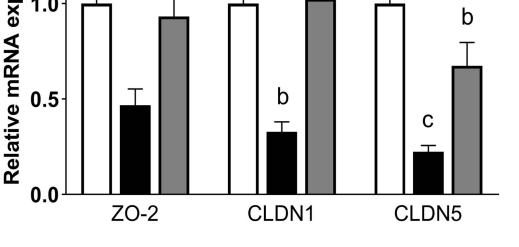

FIGURE 1 | Effect of dietary xylooligosaccharide supplementation on ileal barrier of laying hens fed oxidized fish oil. (A,B) Show the secretory immunoglobulin A ( $\operatorname{sig} A)$ content of ileal mucosal and the endotoxin concentration in serum, respectively. (C) Shows the gene expressions of zona occludens-2 (ZO2), claudin-1 (CLDN1), and claudin-5 (CLDN5) in the ileal mucosa. Data are presented as the means $\pm S E M(n=8)$. FFO, fresh fish oil diet; OFO, oxidized fish oil diet; OFO/XOS 200 , oxidized fish oil diet $+200 \mathrm{mg} / \mathrm{kg}$ of xylooligosaccharide; OFO/XOS 400 , oxidized fish oil diet $+400 \mathrm{mg} / \mathrm{kg}$ of xylooligosaccharide. Bars with no common letter indicate statistical differences among treatments $(P<0.05)$.
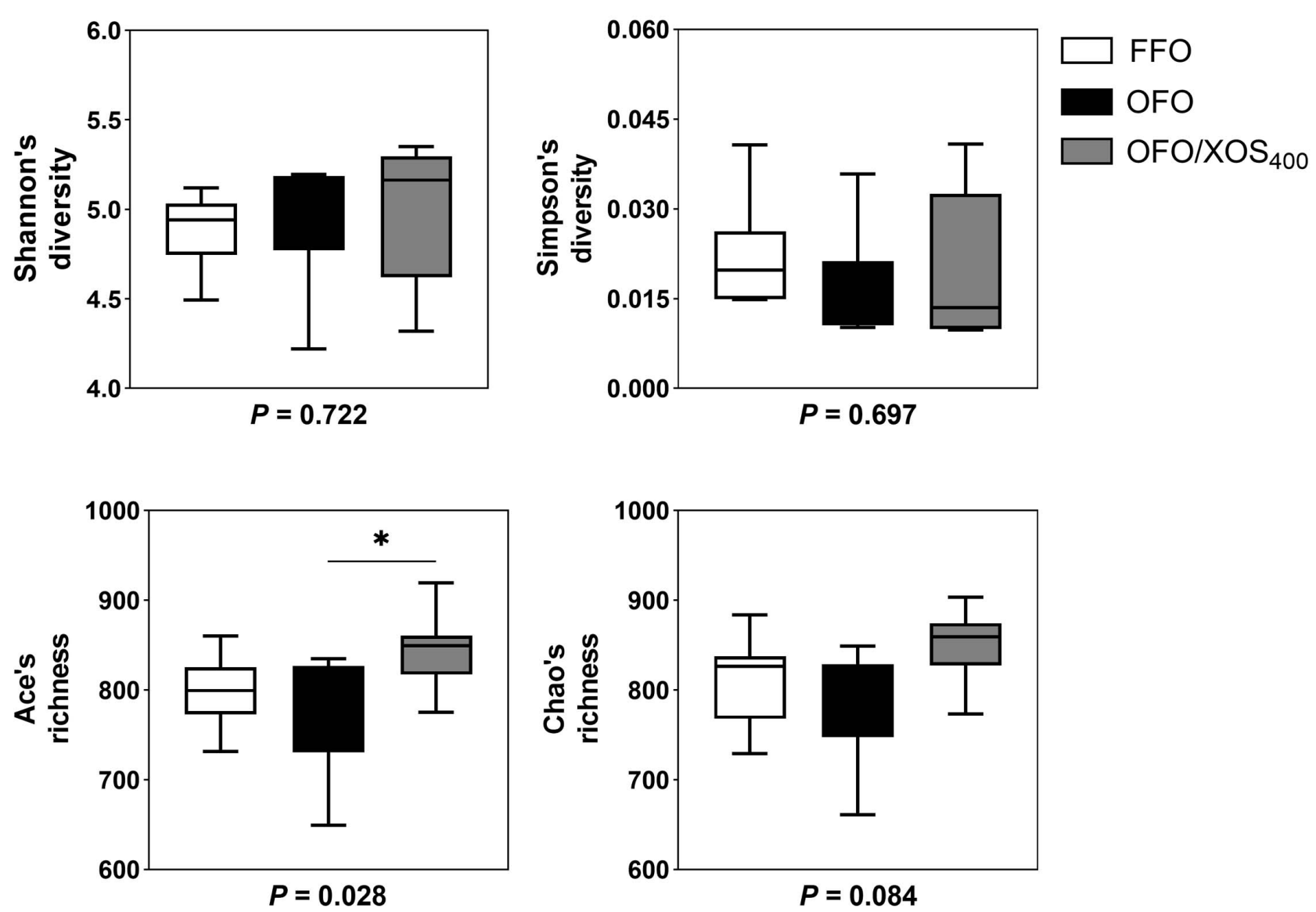

FIGURE 2 | Influence of xylooligosaccharide on cecal bacterial alpha diversity of laying hens fed oxidized fish oil. Four different alpha diversity metrics, Shannon and Simpson diversity indices, and Ace and Chao richness estimators, were compared among three groups. FFO, fresh fish oil diet; OFO, oxidized fish oil diet; $\mathrm{OFO} / \mathrm{XOS}_{400}$, oxidized fish oil diet $+400 \mathrm{mg} / \mathrm{kg}$ xylooligosaccharide. P-values given below each boxplot were estimated by Duncan's multiple range test. * means $P<0.05$.

XOS addition partially reversed these changes found in the OFO group. Within Firmicutes, the majority belonged to the classes Clostridia, Bacilli, and Negativicutes (Figure 4B).
At the family level, the cecal microbiota of layers was dominated by Bacteroidaceae, Rikenellaceae, Ruminococcaceae, and Lachnospiraceae (Figure 4C). OFO treatment led to a 
A

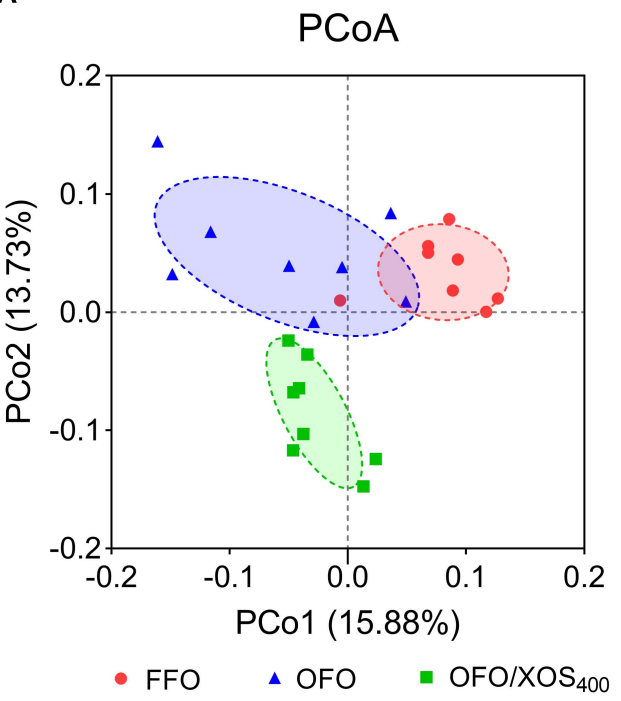

B

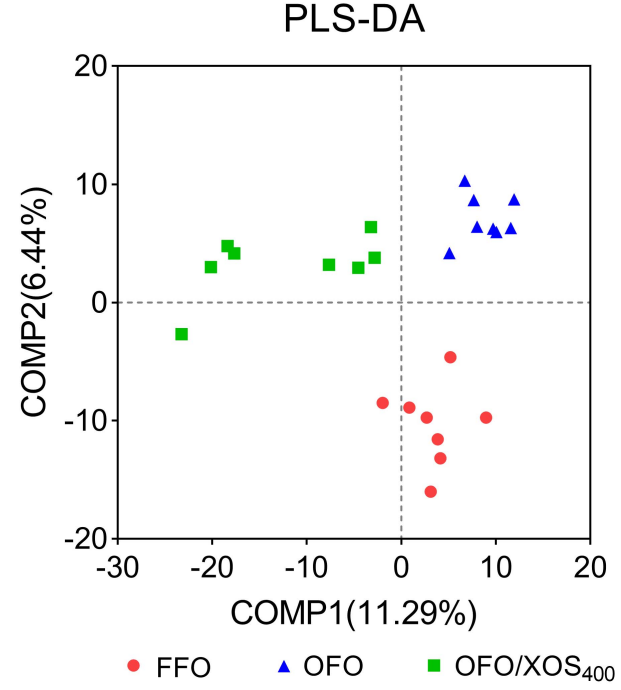

FIGURE 3 | Beta-diversity analysis of cecal microbiota among three groups. (A) Principal coordinate analysis (PCoA) based on unweighted UniFrac distance calculated from OTU abundance matrix. The horizontal axis represents the first principal coordinate, and the vertical axis means the second one. (B) Partial least squares discriminant analysis (PLS-DA). The horizontal axis represents the first component of PLS-DA, and the vertical axis means the second one. The percentages in parentheses represent the explanatory values of the principal coordinates or the components for the difference in sample composition. FFO (red circles), fresh fish oil diet; OFO (blue triangles), oxidized fish oil diet; OFO/XOS 400 (green rectangles), oxidized fish oil diet + $400 \mathrm{mg} / \mathrm{kg}$ xylooligosaccharide.

reduced abundance of Ruminococcaceae with an increased abundance of Rikenellaceae compared with the FFO group, whereas dietary XOS supplementation increased the abundance of Ruminococcaceae to some extent. Genus level analysis showed that the Bacteroides and Rikenellaceae_RC9_gut_group accounted for the largest proportion of the community (Figure 4D). The abundance of Rikenellaceae_RC9_gut_group was increased in layers fed OFO but diminished with XOS addition.

LEfSe analysis was applied to identify the significant differentially abundant OTUs for the entire microbiota at levels from phylum to genus (LDA > 2.0). As shown in Figure 5, the cecal microbiota in the FFO group was enriched with Streptococcaceae (Streptococcus), Butyricicoccus, and Ruminiclostridium_5 as well as Elusimicrobia and its derivatives (Elusimicrobia, Elusimicrobiales, Elusimicrobiaceae, and Elusimicrobium). Birds in the OFO group exhibited increased abundances of family Peptostreptococcaceae and genera Faecalibacterium, Butyricimonas, and Faecalicoccus. In the OFO/XOS 400 group, LEfSe highlights substantial bacterial members enriched in the cecum, some of which accounted for greater proportions and had higher LDA scores such as phylum Verrucomicrobia and its derivatives (Verrucomicrobiae, Verrucomicrobiales, Verrucomicrobiaceae, and Akkermansia) in addition to the genera Paraprevotella, Prevotella_9, and Oscillospira. The Wilcoxon rank-sum test was further performed to reveal the differences in the microbial composition (Table 5). Microbiota in the cecum of birds fed OFO presented lower $(P<0.05)$ abundances of Verrucomicrobia (Akkermansia), Elusimicrobia (Elusimicrobium), and Ruminococcaceae_UCG-008 when compared with those in the FFO group. Besides, OFO treatment triggered a higher abundance of Fusobacteria $(P<0.05)$ than those in FFO group. There were elevations $(P<0.05)$ in the abundances of Verrucomicrobia (Verrucomicrobiaceae, Akkermansia), Paraprevotella, Oscillospira, and Ruminococcaceae_UCG-013 following XOS addition, which also decreased $(P<0.05)$ the abundance of Erysipelatoclostridium compared with that in the OFO group.

\section{Functional Prediction of Gut Microbiota}

Alterations in the presumptive functions of the cecal microbiota of layers were evaluated by PICRUSt analysis. Metagenomic prediction based on COG categories (Supplementary Figure S2A) revealed that the functional pathways enriched within the microbiota were those corresponding to carbohydrate transport and metabolism, amino acid transport and metabolism, general function prediction only, transcription, replication, recombination and repair, cell wall/membrane/envelope biogenesis, as well as translation, ribosomal structure, and biogenesis. In terms of the KEGG prediction (Supplementary Figure S2B), the microbiota represented in the cecum were mainly associated with the following biological processes: membrane transport, carbohydrate metabolism, amino acid metabolism, replication and repair, energy metabolism, and translation. However, there were no differences $(P>0.10)$ noted in the predicted pathways of the microbiota among all groups.

\section{Correlation Between Phylotypes and Phenotypes}

Pearson correlation analysis was conducted to estimate the association between phylotypes in cecal microbiota and 
A

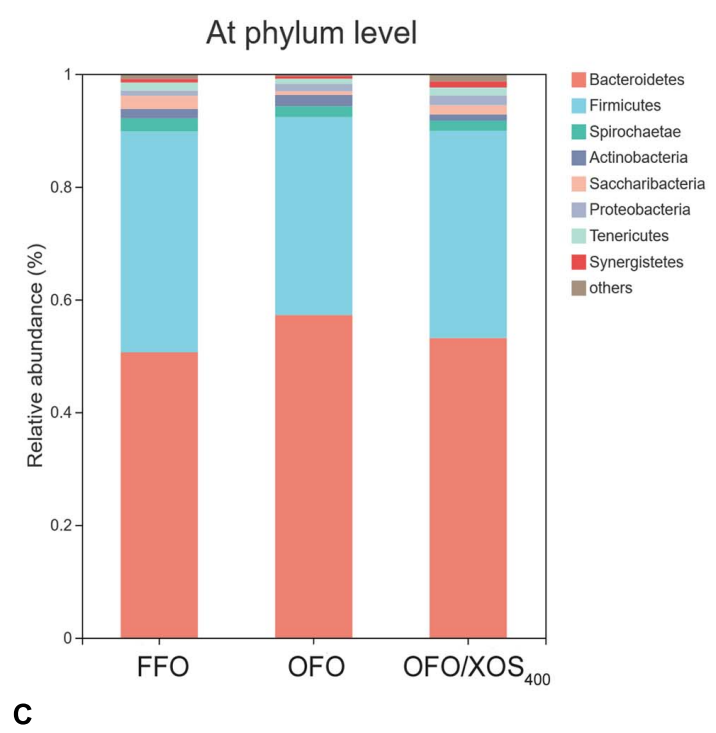

。

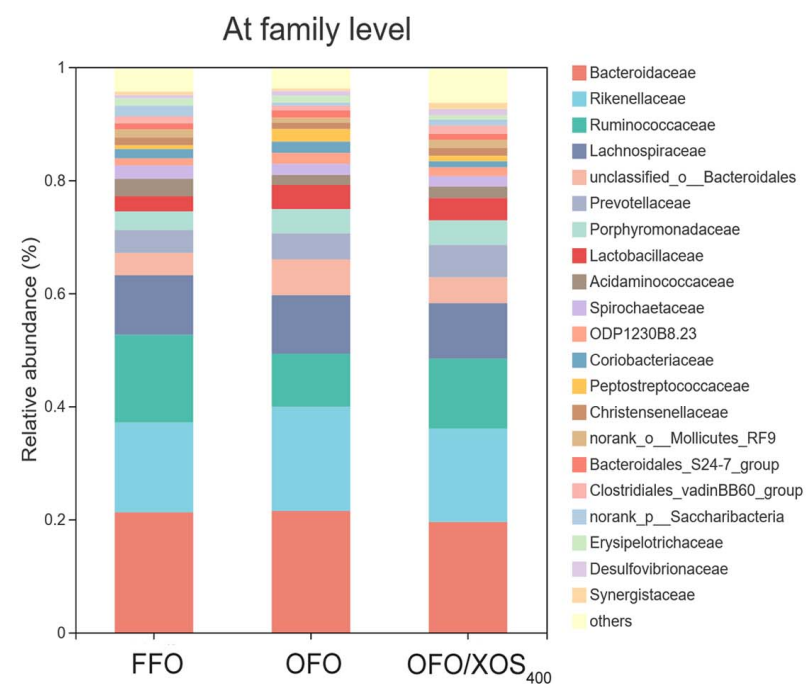

B

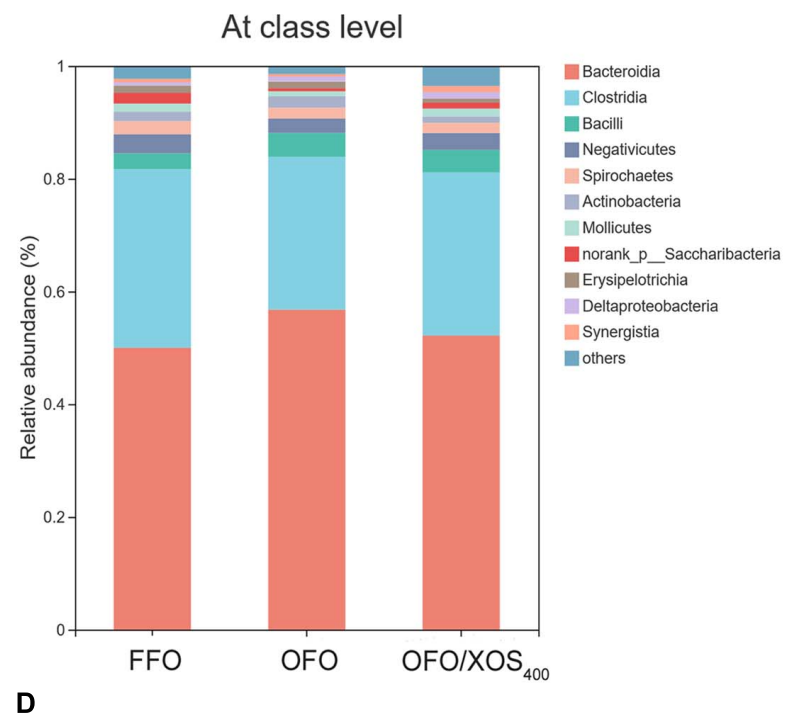

At genus level

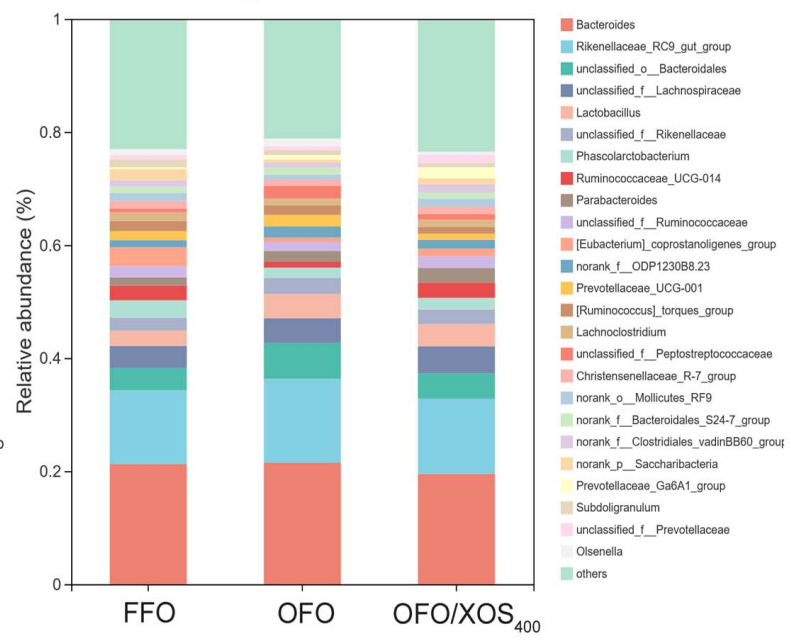

FIGURE 4 | Effect of dietary xylooligosaccharide supplementation on cecal microbial composition at different taxonomic levels of laying hens fed oxidized fish oil (A) at phylum level, (B) at class level, (C) at family level, and (D) at genus level. Those abundance below $1 \%$ were classified as "others." The relative abundance value shown in the plot represent the average of eight samples in each group. FFO, fresh fish oil diet; OFO, oxidized fish oil diet; OFO/XOS 400 , oxidized fish oil diet $+400 \mathrm{mg} / \mathrm{kg}$ xylooligosaccharide.

phenotypes of layers. As shown in Figure 6A, the abundance of phylum Verrucomicrobia was positively correlated $(P<0.05)$ with ileal $\mathrm{VH}$ and the expression of CLDN1 in ileal mucosa. In contrast, the abundance of phylum Fusobacteria showed negative correlation $(P<0.05)$ with the VCR of ileum and sIgA content in ileal mucosa. Comparatively, Elusimicrobia was positively correlated with the expression of CLDN1 $(P<0.05)$ and CLDN5 $(P<0.01)$, but negatively correlated $(P<0.05)$ with the endotoxin concentration in serum. In terms of the cecal microbiota at genus level, the abundances of Erysipelatoclostridium, Turicibacter, and an unclassified member of Peptostreptococcaceae were negatively correlated $(P<0.05)$ with ileal VH, the VCR, GCN, and sIgA concentration, as well as the expression of ileal mucosal ZO2, CLDAN1, and CLDN5 (Figure 6B). Conversely, the abundances of Oscillospira, Paraprevotella, and R. UCG-008 were positively correlated $(P<0.05)$ with ileal VH and/or GCN. In addition, the relative abundance of Akkermansia was also positively associated $(P<0.05)$ with ileal $\mathrm{VH}$ and the expression of $\mathrm{ZO} 2$ and CLDN1, whereas negative correlations $(P<0.05)$ were detected between Butyricimonas and ileal mucosal expression of CLDN1, CLDN5, as well as sIgA concentration. In contrast, the abundance of Elusimicrobium was positively correlated with ileal mucosal CLDN1 $(P<0.05)$ and CLDN5 $(P<0.01)$ expression, but negatively associated $(P<0.05)$ with the endotoxin content in serum. 


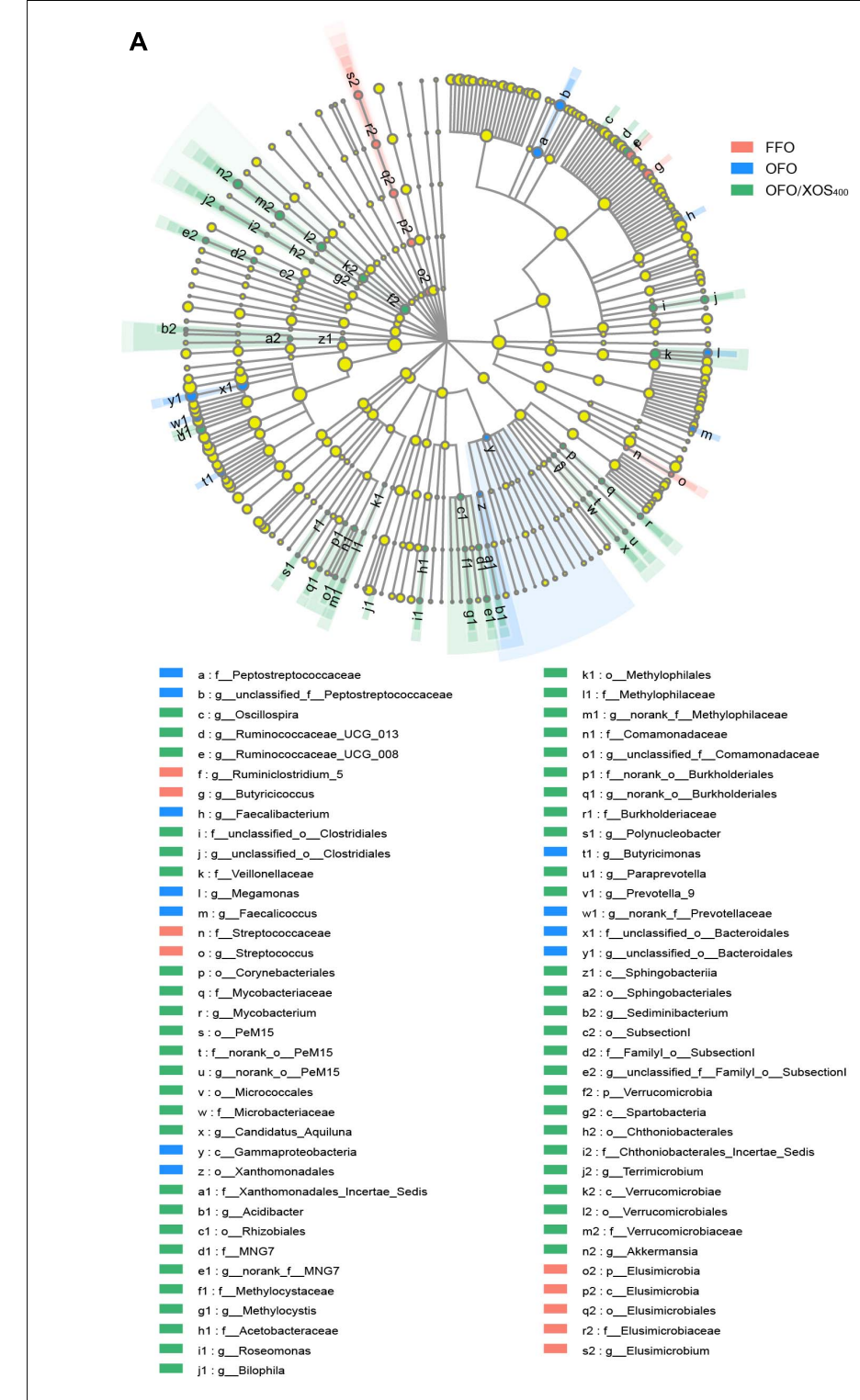

B

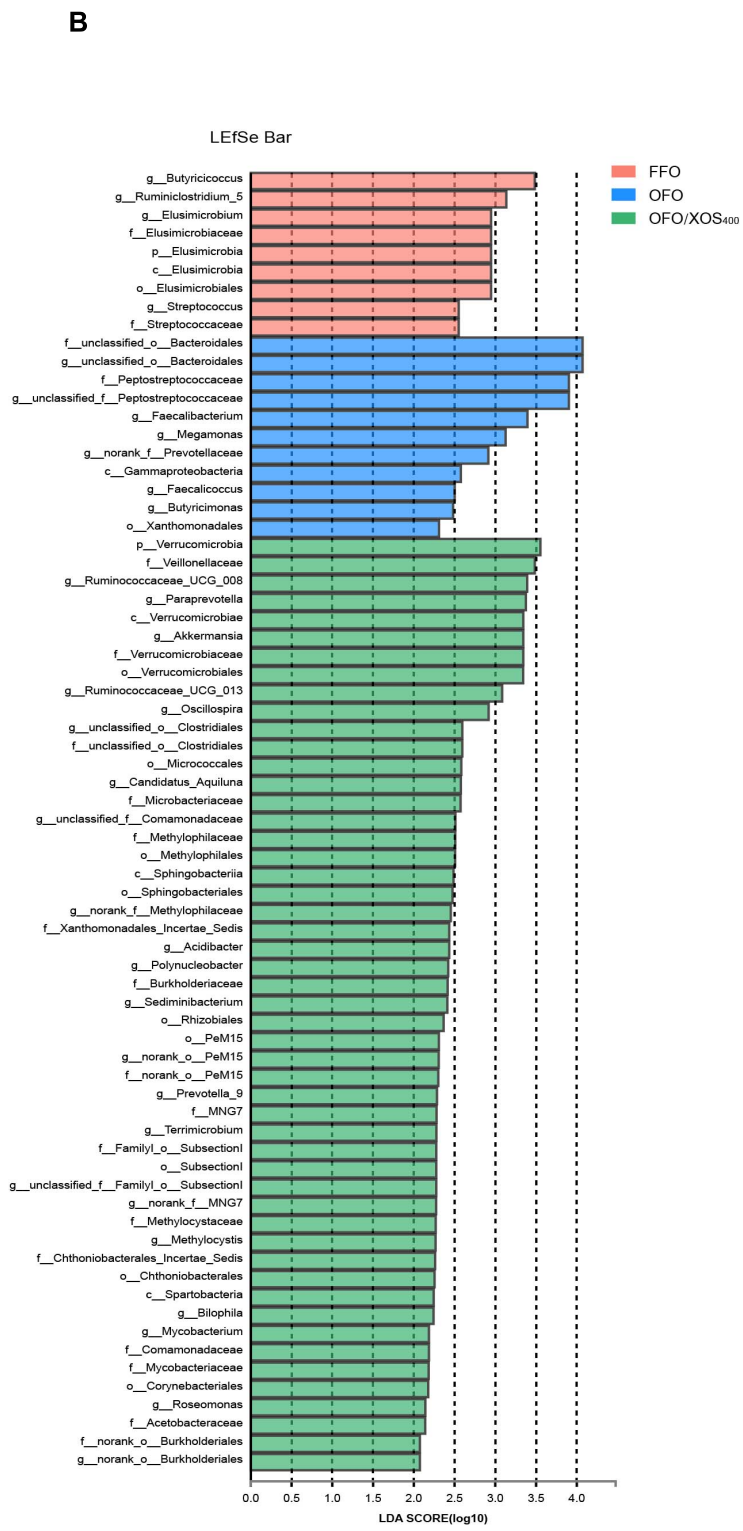

FIGURE 5 | LEfSe identified the most differentially abundant taxa enriched in cecal microbiota among groups FFO (fresh fish oil diet), OFO (oxidized fish oil diet), and OFO/XOS 400 (oxidized fish oil diet $+400 \mathrm{mg} / \mathrm{kg}$ xylooligosaccharide). (A) Cladogram generated from LEfSe analysis, where red, blue, and green circles represent taxa of greater abundance of hens in the FFO, OFO, and OFO/XOS 400 groups, respectively. Yellow circles mean non-significant differences. The diameters of the circles are proportional to the taxon's abundance. (B) Histogram of the LDA scores computed for features differentially abundant among FFO (red bars), OFO (blue bars), and OFO/XOS 400 (green bars) groups. Species with significant difference that have an LDA score greater than 2.0 are presented. The length of the histogram represents the LDA score, which can be interpreted as the effect size of each differentially abundant feature.

\section{DISCUSSION}

Previous studies evidenced that supplemental XOS was associated with an improvement on performance of poultry (Min et al., 2016; Ribeiro et al., 2018; Craig et al., 2020). Similarly, poor performance of layers induced by OFO treatment in the current study was relieved following XOS addition, reflected by the improved EP and FCR. Reduced performance in the OFO group may be caused by some secondary lipid peroxidation products in oxidized fish oil, such as $\alpha$ and $\beta$-unsaturated hydroxyaldehydes
(Tan et al., 2019). These toxicants are highly toxic and readily absorbed, which can damage the intestinal proliferative cells and functional cells of birds (Dibner et al., 1996). Intestinal morphology is considered to be relevant to animal performance because an expanded absorptive surface area means greater digestion and absorption (Montagne et al., 2003; Yamauchi, 2007). In concert with previous studies (De Maesschalck et al., 2015; Min et al., 2016; Ding et al., 2018), the current study showed that hens fed OFO diet with XOS supplementation (400 mg/kg) exhibited higher ileal VH and VCR, which might 
be due to its role in stimulating butyrate-producing microbes to produce butyrate to fuel intestinal epithelial cells (Liu et al., 2018). In order to elucidate the potential mechanism underlying the improved EP and FCR and improved ileal morphology following XOS addition, the variation of ileal mucosal barrier was further investigated.

The intestinal mucosal barrier is the first innate line of defense against pathogen invasion via the cooperation of epithelial cells, mucus layers, sIgA, and tight junctions (Derrien et al., 2017; Ma et al., 2018; Xie et al., 2019b). Goblet cells are differentiated epithelial cells that play a vital role in intestinal mucosal barrier maintenance via the secretion of mucus (Knoop and Newberry, 2018). In the current study, supplemental XOS (400 mg/kg) led to an increased GCN in the ileum of layers, suggesting a possible reinforcement of mucus shield that could be helpful in protecting against intestinal mucosal injury. SIgA secreted by epithelial cells is the principal immunoglobulin in the intestinal mucosal mucus, which was viewed as a key indicator of intestinal immune system (Belkaid and Hand, 2014; Azzam et al., 2017). Similar to a previous study (Min et al., 2016), our results showed that the reduced sIgA content in ileal mucosa induced by OFO was reversed by XOS supplementation at $400 \mathrm{mg} / \mathrm{kg}$, implying an improvement of intestinal immune function in laying hens. Furthermore, tight junction proteins are intercellular junctional molecules that could control intestinal permeability thus thwarting the entry of pathogen and toxins (Barekatain et al., 2019). Therefore, reductions in mRNA expression of CLDN1 and CLDN5 in ileal mucosa observed in birds fed OFO provided a clue that intestinal mucosal barrier was impaired by OFO treatment. In support of this view, hens receiving the OFO diet without XOS addition presented a higher serum endotoxin level which served as an indicator of intestinal barrier damage (Mani et al., 2012). This increase in the concentration of serum endotoxin in the
OFO group might be due to the lipid hydroperoxides generated from the oxidation of fish oil, which could induce a loss of membrane integrity, dysregulation of membrane transport, and alteration in membrane permeability (Wijeratne and Cuppett, 2006; Dasilva et al., 2017). However, in the current study, XOS addition $(400 \mathrm{mg} / \mathrm{kg}$ ) resulted in upregulated ileal mucosal gene expression of CLDN1 and CLDN5 along with reduced serum endotoxin level of layers fed OFO diet, bringing an evidence for the alleviation of OFO-induced intestinal mucosal impairment of laying hens. Similar results were also described by some previous studies, in which dietary XOS supplementation protected intestinal mucosal barrier against challenge in poultry (Eeckhaut et al., 2008; Pourabedin et al., 2017).

The cecum of poultry contains the most detailed information regarding gut microbiota and is the key region for bacterial fermentation of oligosaccharides (Pourabedin and Zhao, 2015). Given the essential roles of cecal microbiota in mediating the manipulation of intestinal barrier by dietary intervention (Wang et al., 2019a; Miao et al., 2020), the alteration of cecal microbiota in the current study was further analyzed. The high species diversity reflects a more stable microbiota community and prevents the colonization of pathogens, thus benefiting to productivity of the host bird (Janczyk et al., 2009; Han et al., 2017). Herein, results of alpha diversity analysis showed that XOS addition $(400 \mathrm{mg} / \mathrm{kg})$ increased cecal microbial richness in layers fed OFO diet, which was consistent with previous studies (Ou et al., 2016; Han et al., 2020). In addition, data from analysis of beta diversity showed significant clustering according to experimental groups, demonstrating that cecal microbial community structure was both affected by $\mathrm{OFO}$ diets with and without XOS addition. Notably, samples in the FFO and $\mathrm{OFO} / \mathrm{XOS}_{400}$ groups were also clustered separately (Supplementary Table S1). We thus speculated that XOS

TABLE 5 | Differences in relative abundance (\%) of cecal microbiota at phylum and genus in laying hens in different groups ${ }^{1}$.

\begin{tabular}{|c|c|c|c|c|c|}
\hline \multirow[t]{2}{*}{ Taxonomic group 2} & \multicolumn{3}{|c|}{ Experimental treatment ${ }^{3}$} & \multicolumn{2}{|c|}{$P$-value } \\
\hline & FFO & OFO & $\mathrm{OFO}_{\mathrm{XOS}} 400$ & FFO vs. OFO & OFO vs. OFO/XOS 400 \\
\hline \multicolumn{6}{|l|}{ Phylum } \\
\hline Verrucomicrobia & $0.439 \pm 0.250$ & $0.096 \pm 0.064$ & $0.726 \pm 0.498$ & 0.024 & 0.046 \\
\hline Elusimicrobia & $0.220 \pm 0.124$ & $0.057 \pm 0.035$ & $0.115 \pm 0.078$ & 0.002 & 0.155 \\
\hline Fusobacteria & $0.003 \pm 0.003$ & $0.027 \pm 0.025$ & $0.004 \pm 0.003$ & 0.046 & 0.188 \\
\hline \multicolumn{6}{|l|}{ Genus } \\
\hline Akkermansia & $0.028 \pm 0.014$ & $0.001 \pm 0.001$ & $0.383 \pm 0.152$ & 0.018 & 0.013 \\
\hline Paraprevotella & $0.437 \pm 0.470$ & $0.223 \pm 0.248$ & $0.666 \pm 0.349$ & 0.103 & 0.018 \\
\hline Oscillospira & $0.105 \pm 0.084$ & $0.040 \pm 0.044$ & $0.186 \pm 0.104$ & 0.058 & 0.003 \\
\hline Erysipelatoclostridium & $0.162 \pm 0.114$ & $0.356 \pm 0.220$ & $0.143 \pm 0.082$ & 0.074 & 0.027 \\
\hline Elusimicrobium & $0.220 \pm 0.124$ & $0.057 \pm 0.035$ & $0.078 \pm 0.055$ & 0.002 & 0.481 \\
\hline Butyricimonas & $0.093 \pm 0.052$ & $0.135 \pm 0.057$ & $0.082 \pm 0.031$ & 0.045 & 0.027 \\
\hline R. UCG-008 & $0.393 \pm 0.239$ & $0.159 \pm 0.184$ & $0.605 \pm 0.690$ & 0.031 & 0.052 \\
\hline R. UCG-013 & $0.352 \pm 0.172$ & $0.222 \pm 0.134$ & $0.451 \pm 0.300$ & 0.141 & 0.014 \\
\hline P. unclassified & $0.668 \pm 0.797$ & $2.246 \pm 1.213$ & $0.960 \pm 0.423$ & 0.014 & 0.052 \\
\hline
\end{tabular}

${ }^{1}$ Data are presented as the means \pm standard deviation $(n=8)$.

${ }^{2}$ R. UCG-008, Ruminococcaceae_UCG-008; R. UCG-013, Ruminococcaceae_UCG-013; P. unclassified, Peptostreptococcaceae unclassified.

${ }^{3} \mathrm{FFO}$, fresh fish oil diet; OFO, oxidized fish oil diet; OFO/XOS 400 , oxidized fish oil diet $+400 \mathrm{mg} / \mathrm{kg}$ xylooligosaccharide. 


\section{A}
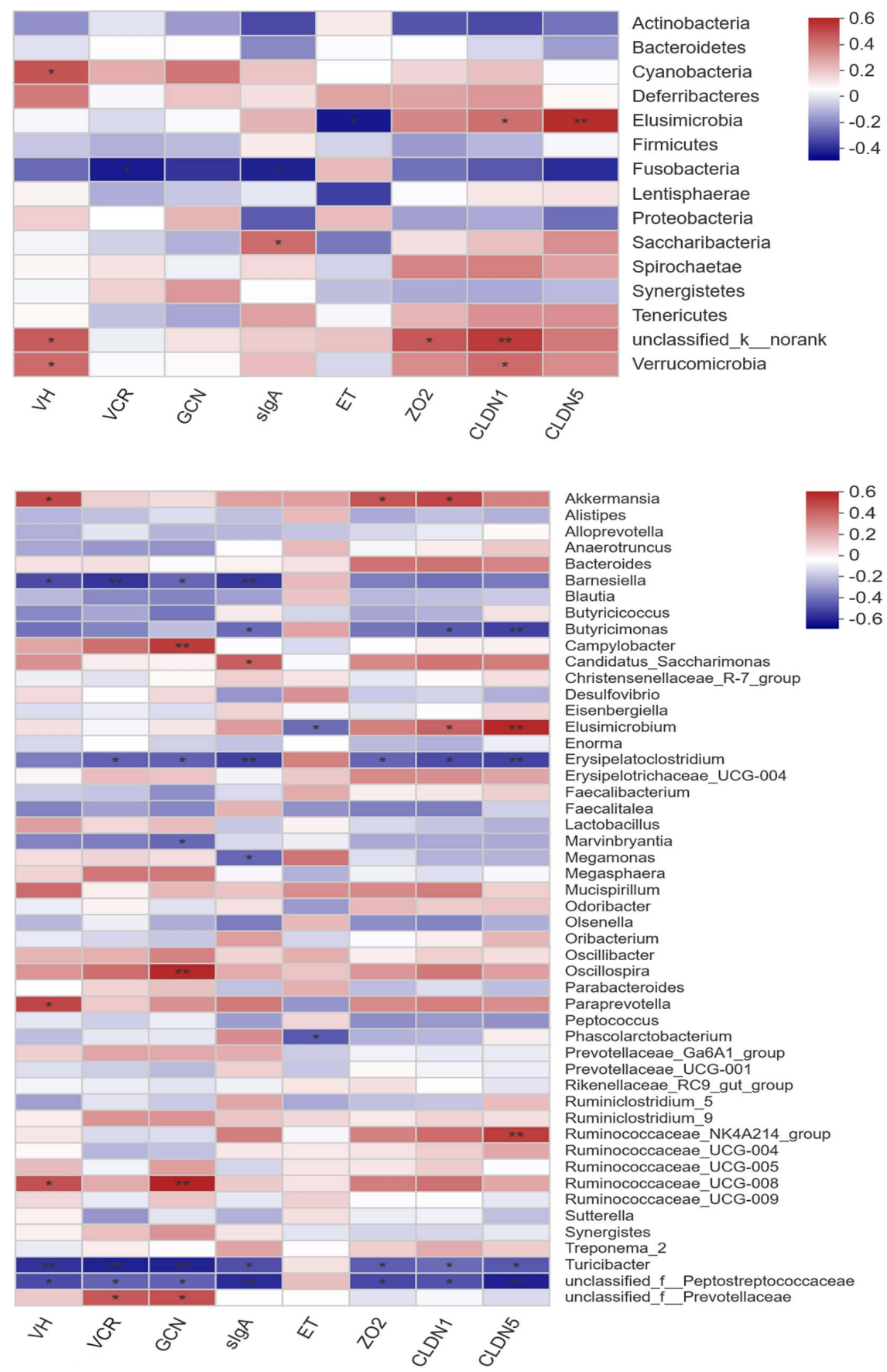

FIGURE 6 | Correlation matrix between key phylotypes (A at phylum level, B at genus level) and phenotypes in laying hens. VH, villus height; VCR, the villus height-to-crypt depth ratio; GCN, goblet cell number; slgA, secretory immunoglobulin A; ET, endotoxin; ZO2, zona occludens-2; CLDN1, claudin-1; CLDN5, claudin-5. The depth of colors ranging from blue to red represents the magnitude of correlation. The OTUs were organized according to their Pearson correlation coefficient. Significant correlations are noted by ${ }^{\star} 0.01<P \leq 0.05,{ }^{* \star} 0.001<P \leq 0.01$.

addition modulated cecal microbiota of layers and not through restoring the bacterial structure that was disturbed by OFO treatment. Changes in microbial composition and some specific taxon in the current study were further analyzed.
Data showed that the cecal microbial composition of birds fed OFO was shifted at the phylum level by favoring Bacteroidetes at the expense of Firmicutes, whereas XOS addition increased the abundance of Firmicutes and reduced the abundance of 
Bacteroidetes, which is consistent with our previous study (Zhou et al., 2020). The abundance of Firmicutes has been proven to be positively correlated with energy and nutrient absorption, whereas an increase in fecal Bacteroidetes is associated with poor nutrient digestibility (Turnbaugh et al., 2006; Jumpertz et al., 2011). Therefore, the increased abundance of Firmicutes and the reduced abundance of Bacteroidetes in cecum may contribute to the melioration of OFO-induced poor FCR of layers following $\mathrm{XOS}$ addition. Additionally, XOS addition reversed a reduction in the abundance of Ruminococcaceae and an increase in the abundance of Rikenellaceae (Rikenellaceae_RC9_gut_group) caused by OFO treatment. As a member of SCFA producers, Ruminococcaceae has been illustrated to be responsible for the degradation of various polysaccharides and fibers (Hooda et al., 2012), leading to a positive correlation between Ruminococcaceae and feed efficiency in chickens (Torok et al., 2011). Besides, it was also reported that the abundance of Ruminococcaceae significantly and inversely correlated with intestinal permeability, thus benefiting gut barrier function (Leclercq et al., 2014). Rikenellaceae has been referred as indicators of inflammation that can recognize endotoxemia markers in animals (Zhai et al., 2017; Wang et al., 2018). Therefore, the altered abundance of Rikenellaceae among groups in the present study might be related to the different serum endotoxin content.

Excluding the dominant bacteria, some other representative species were identified as biomarkers to distinguish cecal microbiota of layers among groups. In the OFO group, LefSe highlights the greater differential abundances of family Peptostreptococcaceae, and genera Faecalibacterium, Butyricimonas, and Faecalicoccus. Peptostreptococcaceae in the human gut represented a maleficent bacterial group closely related to fatty liver and ulcerative colitis (Jiang et al., 2015; Lavelle et al., 2015). Faecalibacterium is a well-known colonizer in the cecum of poultry that participated in the intestinal proinflammatory response stimulated by recycled poultry litter (Shanmugasundaram et al., 2011; Wang et al., 2016). Butyricimonas and Faecalicoccus were rarely mentioned in poultry, as they were recently described and classified as novel genera (Kanno et al., 2015; Forbes et al., 2018). Nevertheless, Butyricimonas exhibited negative association with intestinal mucosal sIgA content and expression of CLDN1 and CLDN5 in the current study. The Wilcoxon rank-sum test further revealed some differential species between OFO and FFO treatments. For example, OFO treatment triggered a significantly increased Fusobacteria, which possesses virulence characteristics that can adhere and invade into host epithelial cells to elicit proinflammatory response (Strauss et al., 2011; Kostic et al., 2012). Consistently, the negative correlations between Fusobacteria and ileal VCR and sIgA content were observed in the current study. These increased potential pathogens mentioned above might contribute to the impairment of intestinal mucosal barrier induced by OFO treatment. In addition, Akkermansia is a strict anaerobe and uses the mucin in gut mucus as the sole source of carbon and nitrogen elements (Derrien et al., 2017). Some members of Elusimicrobia were characterized by small size with thin and fragile outer envelope, making them sensitive to adverse conditions (Zheng et al.,
2015; Martinez-Fernandez et al., 2019). Thus, the reductions of Akkermansia and Elusimicrobia (Elusimicrobium) in the OFO group might be the consequences of the impaired intestinal mucosal barrier, including the possible reduction of mucus generation. Taken together, OFO diet exactly caused a dysbiosis in cecal microbiota of laying hens, which was probably involved in the impairment of intestinal mucosal barrier though it was hard to determine which came first, the microbiota dysbiosis or an intestinal mucosal damage.

Despite the fact that it is hard to distinguish whether dysbiosis of the cecal microbiota is the cause or consequence of intestinal mucosa damage, many salutary effects of microbiota modulation on gut mucosal barrier by dietary intervention have been already proven in poultry (Pourabedin et al., 2017; Wang et al., 2019b). As a kind of typical prebiotic, XOS has been reported to be beneficial to the health and performance of birds through modulating the gut microbiota (De Maesschalck et al., 2015; Ding et al., 2018). Herein, XOS addition (400 mg/kg) enriched the abundances of Verrucomicrobia (Akkermansia), Paraprevotella, Prevotella_9, and Oscillospira in the cecum of layers. A previous publication classified that some members of Verrucomicrobia were performance-linked species in cecum of chickens (Torok et al., 2011), of which Akkermansia can be served as the first and the most representative one (Zhang et al., 2019). It was reported that Akkermansia spp. not only participate in mucosal immune regulation but also improve the integrity of the intestinal epithelial cells and the thickness of the mucus layer, thus enhancing intestinal mucosal barrier (Everard et al., 2013; Reunanen et al., 2015). Consistently, our results showed that abundance of Akkermansia was positively associated with ileal $\mathrm{VH}$ and the expression of ZO2 and CLDN1. Paraprevotella and Prevotella enriched by XOS treatment were also described by a recent research (Fei et al., 2020), in which they were associated with butyrate generation in the gut because of their capability to degrade cellulose and polysaccharides, which was favorable to FCR of animals fed plant-based diets (Gorvitovskaia et al., 2016; Shah et al., 2018). Besides, Prevotella abundance was also associated with a higher production of luminal sIgA, implying its role in intestinal immune regulation (Mach et al., 2015). Similarly, Oscillospira, a predominant genus in chicken cecal microbiota (Wang et al., 2016), was proved to be negatively correlated with intestinal inflammatory diseases (Gophna et al., 2017). Results of Wilcoxon rank-sum test between OFO and $\mathrm{OFO} / \mathrm{XOS}_{400}$ groups basically support the alterations of cecal microbiota revealed by LefSe analysis. Furthermore, a significant reduction of the abundance of Erysipelatoclostridium was observed in $\mathrm{OFO} / \mathrm{XOS}_{400}$ group compared to that in the OFO group. Erysipelatoclostridium was considered as an opportunistic pathogen (Shao et al., 2017) that exhibited negative associations with ileal VH, VCR, ileal mucosal sIgA content, and expression of ZO2, CLDN1, and CLDN5 in the current study. To sum up, alterations of these bacterial phylotypes suggested that the cecal microbiota of layers fed with XOS became more favorable in protecting intestinal mucosal barrier from impairments by $\mathrm{OFO}$ and further improving production performance. Interestingly, only little bacteria altered by OFO treatment was reversed following XOS addition (400 $\mathrm{mg} / \mathrm{kg}$ ) in layers, which supports 
our hypothesis that XOS addition regulated OFO-induced gut microbiota dysbiosis through remodeling rather than restoring the microbial composition. Further studies are needed to elucidate the potential mechanism.

\section{CONCLUSION}

Supplemental XOS attenuated OFO-induced impairment in production performance and fortified ileal morphology by alleviating intestinal mucosal barrier dysfunction in laying hens, which could be associated to the increase in microbial richness and remodeling of microbial composition. The current study would expand our knowledge concerning the roles of gut microbiota in mediating the manipulation of intestinal mucosal barrier by dietary intervention and can help to improve the efficiency in applying XOS in chicken production.

\section{DATA AVAILABILITY STATEMENT}

The datasets presented in this study can be found in online repositories. The names of the repository/repositories and accession number(s) can be found in the article/ Supplementary Material.

\section{ETHICS STATEMENT}

The animal study was reviewed and approved by the Animal Care and Use Committee of the Feed Research Institute of

\section{REFERENCES}

Alam, A., and Neish, A. (2018). Role of gut microbiota in intestinal wound healing and barrier function. Tissue Barriers 6, 1-22.

Azzam, M. M. M., Dong, X. Y., and Zou, X. T. (2017). Effect of dietary threonine on laying performance and intestinal immunity of laying hens fed low-crudeprotein diets during the peak production period. J. Anim. Physiol. Anim. Nutr. 101, e55-e66.

Barekatain, R., Chrystal, P. V., Howarth, G. S., Mclaughlan, C. J., Gilani, S., and Nattrass, G. S. (2019). Performance, intestinal permeability, and gene expression of selected tight junction proteins in broiler chickens fed reduced protein diets supplemented with arginine, glutamine, and glycine subjected to a leaky gut model. Poult. Sci. 98, 6761-6771. doi: 10.3382/ps/pez393

Belkaid, Y., and Hand, T. (2014). Role of the microbiota in immunity and inflammation. Cell 157, 121-141. doi: 10.1016/j.cell.2014.03.011

Christensen, E. G., Licht, T. R., Leser, T. D., and Bahl, M. I. (2014). Dietary xylo-oligosaccharide stimulates intestinal Bifidobacteria and Lactobacilli but has limited effect on intestinal integrity in rats. BMC Res. Notes 7:660. doi: 10.1186/1756-0500-7-660

Craig, A. D., Khattak, F., Hastie, P., Bedford, M. R., and Olukosi, O. A. (2020). Xylanase and xylo-oligosaccharide prebiotic improve the growth performance and concentration of potentially prebiotic oligosaccharides in the ileum of broiler chickens. Br. Poult. Sci. 61, 70-78. doi: 10.1080/00071668.2019.1673318

Dasilva, G., Boller, M., Medina, I., and Storch, J. (2017). Relative levels of dietary EPA and DHA impact gastric oxidation and essential fatty acid uptake. J. Nutr. Biochem. 55, 68-75. doi: 10.1016/j.jnutbio.2017.11.007

De Maesschalck, C., Eeckhaut, V., Maertens, L., De-Lange, L., Marchal, L., Nezer, C., et al. (2015). Effects of xylo-oligosaccharides on broiler chicken performance and microbiota. Appl. Environ. Microbiol. 81, 5880-5888. doi: 10.1128/aem. 01616-15 the Chinese Academy of Agricultural Sciences (ACE-CAAS20180915).

\section{AUTHOR CONTRIBUTIONS}

JW and JZ conceived and designed the experiments. JZ performed animal experiments, analyzed the data, and wrote the manuscript. JW assisted with data analysis and manuscript writing. GQ, KQ, SW, YF, and HZ supervised and provided continuous guidance for the experiment. All authors have discussed the results and reviewed the manuscript, read, and approved the final manuscript.

\section{FUNDING}

This study was supported by the Shandong Key Science and Technology Innovation Program (2019JZZY010704), the China Agriculture Research System (CARS-40K12), and the Agricultural Science and Technology Innovation Program (ASTIP) of the Chinese Academy of Agricultural Sciences.

\section{SUPPLEMENTARY MATERIAL}

The Supplementary Material for this article can be found online at: https://www.frontiersin.org/articles/10.3389/fmicb. 2021.635333/full\#supplementary-material

Derrien, M., Belzer, C., and Vos, W. M. D. (2017). Akkermansia muciniphila and its role in regulating host functions. Microb. Pathog. 106, 171-181. doi: 10.1016/j.micpath.2016.02.005

Dibner, J. J., Atwell, C. A., Kitchell, M. L., Shermer, W. D., and Ivey, F. J. (1996). Feeding of oxidized fats to broilers and swine: effects on enterocyte turnover hepatocyte proliferation and the gut associated lymphoid tissue. Anim. Feed Sci. Technol. 62, 1-13. doi: 10.1016/s0377-8401(96)01000-0

Ding, X. M., Li, D. D., Bai, S. P., Wang, J. P., Zeng, Q. F., Su, Z. W., et al. (2018). Effect of dietary xylooligosaccharides on intestinal characteristics, gut microbiota, cecal short-chain fatty acids, and plasma immune parameters of laying hens. Poult. Sci. 97, 874-881. doi: 10.3382/ps/pex372

Dong, X. F., Liu, S., and Tong, J. M. (2018). Comparative effect of dietary soybean oil, fish oil, and coconut oil on performance, egg quality and some blood parameters in laying hens. Poult. Sci. 97, 2460-2472. doi: 10.3382/ps/ pey094

Eeckhaut, V., Van-Immerseel, F., Dewulf, J., Pasmans, F., Haesebrouck, F., Ducatelle, R., et al. (2008). Arabinoxylooligosaccharides from wheat bran inhibit Salmonella colonization in broiler chickens. Poult. Sci. 87, 2329-2334. doi: 10.3382/ps.2008-00193

Everard, A., Belzer, C., Geurts, L., Ouwerkerk, J. P., Druart, C., Bindels, L. B., et al. (2013). Cross-talk between Akkermansia muciniphila and intestinal epithelium controls diet-induced obesity. Proc. Natl. Acad. Sci. U. S. A. 110, 9066-9071. doi: 10.1073/pnas.1219451110

Fei, Y. Q., Wang, Y., Pang, Y. L., Wang, W. Y., Zhu, D., Xie, M. G., et al. (2020). Xylooligosaccharide modulates gut microbiota and alleviates colonic inflammation caused by high fat diet induced obesity. Front. Physiol. 10:1601.

Feng, J., Long, S., Zhang, H., Wu, S., Qi, G., and Wang, J. (2019). Comparative effects of dietary microalgae oil and fish oil on fatty acid composition and sensory quality of table eggs. Poult. Sci. 99, 1734-1743. doi: 10.1016/j.psj.2019. 11.005 
Forbes, J. D., Chen, C. Y., Knox, N. C., Marrie, R. A., El-Gabalawy, H., De Kievit, T., et al. (2018). A comparative study of the gut microbiota in immune-mediated inflammatory diseases-does a common dysbiosis exist? Microbiome 6:221.

Forte, C., Acuti, G., Manuali, E., Proietti, P. C., Pavone, S., Trabalzamarinucci, M., et al. (2016). Effects of two different probiotics on microflora, morphology, and morphometry of gut in organic laying hens. Poult. Sci. 95, 2528-2535. doi: $10.3382 /$ ps/pew164

Gophna, U., Konikoff, T., and Nielsen, H. B. (2017). Oscillospira and related bacteria-from metagenomic species to metabolic features. Environ. Microbiol. 19, 835-841. doi: 10.1111/1462-2920.13658

Gorvitovskaia, A., Holmes, S. P., and Huse, S. M. (2016). Interpreting prevotella and Bacteroides as biomarkers of diet and lifestyle. Microbiome 4:15.

Han, D., Li, Z., Liu, T., Yang, N., Li, Y., He, J., et al. (2020). Prebiotics regulation of intestinal microbiota attenuates cognitive dysfunction induced by surgery stimulation in APP/PS1 mice. Aging Dis. 11, 1029-1045. doi: 10.14336/ad.2020. 0106

Han, Z., Willer, T., Li, L., Pielsticker, C., Rychlik, I., Velge, P., et al. (2017). Influence of the gut microbiota composition on Campylobacter jejuni colonization inchickens. Infect. Immun. 85:e380-17.

Hooda, S., Boler, B. M. V., Serao, M. C. R., Brulc, J. M., Staeger, M. A., Boileau, T. W., et al. (2012). 454 pyrosequencing reveals a shift in fecal microbiota of healthy adult men consuming polydextrose or soluble corn fiber. J. Nutr. 142, 1259-1265. doi: 10.3945/jn.112.158766

International Organization for Standarization [ISO] (2001). Animal and Vegetable Fats and oils-Determination of Peroxide Value. ISO 3960: 2001 (IDT.). Geneva: ISO.

Janczyk, P., Halle, B., and Souffrant, W. B. (2009). Microbial community composition of the crop and ceca contents of laying hens fed diets supplemented with Chlorella vulgaris. Poult. Sci. 88, 2324-2332. doi: 10.3382/ ps.2009-00250

Jiang, W., Wu, N., Wang, X., Chi, Y., Zhang, Y., Qiu, X., et al. (2015). Dysbiosis gut microbiota associated with inflammation and impaired mucosal immune function in intestine of humans with non-alcoholic fatty liver disease. Sci. Rep. 5:8096.

Jordan, D. B., and Kurt, W. (2010). Properties and applications of microbial beta $\mathrm{D}$-xylosidases featuring the catalytically efficient enzyme from Selenomonas ruminantium. J. Appl. Microbiol. Biotechnol. 86, 1647-1658. doi: 10.1007/ s00253-010-2538-y

Jumpertz, R., Le, D. S., Turnbaugh, P. J., Trinidad, C., Bogardus, C., Gordon, J. I., et al. (2011). Energy-balance studies reveal associations between gut microbes, caloric load, and nutrient absorption in humans. Am. J. Clin. Nutr. 94, 58-65. doi: 10.3945/ajcn.110.010132

Kanno, M., Katayama, T., Morita, N., Tamaki, H., Hanada, S., and Kamagata, Y. (2015). Catenisphaera adipataccumulans gen. nov., sp. nov., a member of the family Erysipelotrichaceae isolated from an anaerobic digester. Int. J. Syst. Evol. Microbiol. 65, 805-810. doi: 10.1099/ijs.0.000021

Knoop, K. A., and Newberry, R. D. (2018). Goblet cells: multifaceted players in immunity at mucosal surfaces. Mucosal Immunol. 11, 1551-1557. doi: 10.1038/ s41385-018-0039-y

Kostic, A. D., Gevers, D., Pedamallu, C. S., Michaud, M., Duke, F., Earl, A. M., et al. (2012). Genomic analysis identifies association of Fusobacterium with colorectal carcinoma. Genome Res. 22, 292-298. doi: 10.1101/gr.126573.111

Langille, M. G. I., Zaneveld, J., Caporaso, J. G., McDonald, D., Knights, D., Reyes, J. A., et al. (2013). Predictive functional profiling of microbial communities using $16 \mathrm{~S}$ rRNA marker gene sequences. Nat. Biotechnol. 31, 814-821. doi: $10.1038 /$ nbt. 2676

Lapidot, T., Granit, R., and Kanner, J. (2005). Lipid peroxidation by “free" iron ions and myoglobin as affected by dietary antioxidants in simulated gastric fluids. J. Agric. Food Chem. 53, 3383-3390. doi: 10.1021/jf040402g

Lavelle, A., Lennon, G., O’Sullivan, O., Docherty, N., Balfe, A., Maguire, A., et al. (2015). Spatial variation of the colonic microbiota in patients with ulcerative colitis and control volunteers. Gut 64, 1553-1561. doi: 10.1136/gutjnl-2014307873

Leclercq, S., Matamoros, S., Cani, P. D., Neyrinck, A. M., Jamar, F., Stärkel, P., et al. (2014). Intestinal permeability, gut-bacterial dysbiosis, and behavioral markers of alcohol-dependence severity. Proc. Natl. Acad. Sci. U. S. A. 111, E4485-E4493.
Lin, Y., Li, W. L., Huo, Q. Q., Du, C. H., Wang, Z. X., Yi, B. D., et al. (2018). Effects of xylo-oligosaccharide and flavomycin on the immune function of broiler chickens. PeerJ 6:e4435. doi: 10.7717/peerj.4435

Livak, K. J., and Schmittgen, T. D. (2001). Analysis of relative gene expression data using real-time quantitative PCR and the 2- $\Delta \Delta$ Ct method. Methods 25 , 402-408. doi: 10.1006/meth.2001.1262

Liu, H., Wang, J., He, T., Becker, S., Zhang, G., Li, D., et al. (2018). Butyrate: a double-edged sword for health? Adv. Nutr. 9, 21-29.

Ma, N., Guo, P., Zhang, J., He, T., Kim, S. W., and Zhang, G. (2018). Nutrients mediate intestinal bacteria-mucosal immune crosstalk. Front. Immunol. 9:5.

Mach, N., Berri, M., Estellé, J., Levenez, F., Lemonnier, G., Denis, C., et al. (2015). Early-life establishment of the swine gut microbiome and impact on host phenotypes. Environ. Microbiol. Rep. 7, 554-569. doi: 10.1111/1758-2229. 12285

Maestre, R., Douglass, J. D., Kodukula, S., Medina, I., and Storch, J. (2013). Alterations in the intestinal assimilation of oxidized PUFAs are ameliorated by a polyphenol-rich grape seed extract in an in vitro model and Caco-2 cells. J. Nutr. 143, 295-301. doi: 10.3945/jn.112.160101

Mani, V., Weber, T. E., Baumgard, L. H., and Gabler, N. K. (2012). Growth and development symposium: endotoxin, inflammation, and intestinal function in livestock. J. Anim. Sci. 90, 1452-1465. doi: 10.2527/jas.2011-4627

Martinez-Fernandez, G., Denman, S. E., and McSweeney, C. S. (2019). Sample processing methods impacts on rumen microbiome. Front. Microbiol. 10:861.

Miao, L., Gong, Y., Li, H., Xie, C., Xu, Q., Dong, X., et al. (2020). Alterations in cecal microbiota and intestinal barrier function of laying hens fed on fluoride supplemented diets. Ecotoxicol. Environ. Saf. 193:110372. doi: 10.1016/j.ecoenv. 2020.110372

Min, Y. N., Yang, H. L., Xu, Y. X., and Gao, Y. P. (2016). Effects of dietary supplementation of synbiotics on growth performance, intestinal morphology, sIgA content and antioxidant capacities of broilers. J. Anim. Physiol. Anim. Nutr. 100, 1073-1080. doi: 10.1111/jpn.12479

Montagne, L., Crévieu-Gabriel, I., Toullec, R., and Lallès, J. P. (2003). Influence of dietary protein level and source on the course of protein digestion along the small intestine of the veal calf. J. Dairy Sci. 86, 934-943. doi: 10.3168/jds.s00220302(03)73676-5

National Research and Council (1994). Nutrient Requirements of Poultry: Ninth Revised Edition. Washington, DC: The National Academies Press.

Ou, J. Y., Huang, J. Q., Song, Y., Yao, S. W., Peng, X. C., Wang, M. F., et al. (2016). Feruloylated oligosaccharides from maize bran modulated the gut microbiota in rats. Plant Foods Hum. Nutr. 71, 123-128. doi: 10.1007/s11130-016-0547-4

Pourabedin, M., and Zhao, X. (2015). Prebiotics and gut microbiota in chickens. FEMS Microbiol. Lett. 362:fnv122. doi: 10.1093/femsle/fnv122

Pourabedin, M., Chen, Q. L., Yang, M. M., and Zhao, X. (2017). Mannan- and xylooligosaccharides modulate cecal microbiota and expression of inflammatory related cytokines and reduce cecal Salmonella enteritidis colonization in young chickens. FEMS Microbiol. Ecol. 93:fiw226. doi: 10.1093/femsec/fiw226

Reunanen, J., Kainulainen, V., Huuskonen, L., Ottman, N., Belzer, C., Huhtinen, H., et al. (2015). Akkermansia muciniphila adheres to enterocytes and strengthens the integrity of the epithelial cell layer. Appl. Environ. Microbiol. 81, 3655-3662. doi: 10.1128/aem.04050- 14

Ribeiro, T., Cardoso, V., Ferreira, L. M. A., Lordelo, M. M. S., Coelho, E., Moreira, A. S. P., et al. (2018). Xylo-oligosaccharides display a prebiotic activity when used to supplement wheat or corn-based diets for broilers. Poult. Sci. 97, 4330-4341. doi: 10.3382/ps/pey336

Sánchez de Medina, F., Romero-Calvo, I., Mascaraque, C., and Martínez-Augustin, O. (2014). Intestinal inflammation and mucosal barrier function. Inflamm. Bowel Dis. 20, 2394-2404. doi: 10.1097/mib.0000000000000204

Shah, H., Sami, J., Olli, P., Lars, P., Annina, L., Juhani, V., et al. (2018). Dietary supplementation with yeast hydrolysate in pregnancy influences colostrum yield and gut microbiota of sows and piglets after birth. PLoS One 13:e0197586. doi: 10.1371/journal.pone.0197586

Shanmugasundaram, R., Lilburn, M. S., and Selvaraj, R. K. (2011). Effect of recycled litter on immune cells in the cecal tonsils of chickens. Poult. Sci. 91, 95-100. doi: 10.3382/ps.2011-01800

Shao, T., Shao, L., Li, H., Xie, Z., He, Z., and Wen, C. (2017). Combined signature of the fecal microbiome and metabolome in patients with gout. Front. Microbiol. $8: 268$. 
Strauss, J., Kaplan, G. G., Beck, P. L., Rioux, K., Panaccione, R., Devinney, R., et al. (2011). Invasive potential of gut mucosa-derived Fusobacterium nucleatum positively correlates with IBD status of the host. Inflamm. Bowel Dis. 17, 1971-1978. doi: 10.1002/ibd.21606

Tan, L. L., Rong, D., Yang, Y. Y., and Zhang, B. K. (2019). The effect of oxidized fish oils on growth performance, oxidative status, and intestinal barrier function in broiler chickens. J. Appl. Poult. Res. 28, 31-41. doi: 10.3382/japr/ pfy013

Torok, V. A., Hughes, R. J., Mikkelsen, L. L., Perez-Maldonado, R., and OphelKeller, K. (2011). Identification and characterization of potential performancerelated gut microbiotas in broiler chickens across various feeding trials. Appl. Environ. Microbiol. 77, 5868-5878. doi: 10.1128/aem.00165-11

Turnbaugh, P. J., Ley, R. E., Mahowald, M. A., Vincent, M., Mardis, E. R., and Gordon, J. I. (2006). An obesity-associated gut microbiome with increased capacity for energy harvest. Nature 444, 1027-1031.

Wang, H., Liang, S. S., Li, X. Y., Yang, X. J., Long, F. Y., and Yang, X. (2019a). Effects of encapsulated essential oils and organic acids on laying performance, egg quality, intestinal morphology, barrier function, and microflora count of hens during the early laying period. Poult. Sci. 98, 6751-6760. doi: 10.3382/ps/ pez391

Wang, L., Lilburn, M., and Yu, Z. (2016). Intestinal microbiota of broiler chickens as affected by litter management regimens. Front. Microbiol. 7:593.

Wang, W. W., Jia, H. J., Zhang, H. J., Wang, J., Lv, H. Y., Wu, S. G., et al. (2019b). Supplemental plant extracts from flos ionicerae in combination with baikal skullcap attenuate intestinal disruption and modulate gut microbiota in laying hens challenged by Salmonella pullorum. Front. Microbiol. 10:1681.

Wang, Y., Zhang, H., Zhu, L., Xu, Y., Liu, N., Sun, X., et al. (2018). Dynamic distribution of gut microbiota in goats at different ages and health states. Front. Microbiol. 9:2509.

Wijeratne, S. S. K., and Cuppett, S. L. (2006). Lipid hydroperoxide induced oxidative stress damage and antioxidant enzyme response in caco-2 human colon cells. J. Agric. Food Chem. 54, 4476-4481. doi: 10.1021/jf060475v

Xie, S., Zhao, S., Jiang, L., Lu, L., Yang, Q., and Yu, Q. (2019a). Lactobacillus reuteri stimulates intestinal epithelial proliferation and induces differentiation into goblet cells in young chickens. J. Agric. Food Chem. 67, 13758-13766. doi: 10.1021/acs.jafc.9b06256
Xie, X. L., He, Y. Q., Li, H., Yu, D., Na, L., Sun, T., et al. (2019b). Effects of prebiotics on immunologic indicators and intestinal microbiota structure in perioperative colorectal cancer patients. Nutrition 61, 132-142. doi: 10.1016/j.nut.2018.10. 038

Xing, S. C., Mi, J. D., Chen, J. Y., Hu, J. X., and Liao, X. D. (2019). Metabolic activity of Bacillus coagulans R11 and the health benefits of and potential pathogen inhibition by this species in the intestines of laying hens under lead exposure. Sci. Total Environ. 709:134507. doi: 10.1016/j.scitotenv.2019.134507

Yamauchi, K. E. (2007). Review of a histological intestinal approach to assessing the intestinal function in chickens and pigs. Anim. Sci. J. 78, 356-370. doi: 10.1111/j.1740-0929.2007.00448.x

Zhai, Q. X., Li, T. Q., Yu, L. L., Xiao, Y., Feng, S. S., Wu, J. P., et al. (2017). Effects of subchronic oral toxic metal exposure on the intestinal microbiota of mice. Sci. Bull. 62, 831-840. doi: 10.1016/j.scib.2017.01.031

Zhang, T., Li, Q., Cheng, L., Buch, H., and Zhang, F. (2019). Akkermansia muciniphila is a promising probiotic. Microb. Biotechnol. 12, 1109-1125. doi: 10.1111/1751-7915.13410

Zheng, H., Dietrich, C., Radek, R., and Brune, A. (2015). Endomicrobium proavitum, the first isolate of Endomicrobia class. nov. (phylum Elusimicrobia)an ultramicrobacterium with an unusual cell cycle that fixes nitrogen with a Group IV nitrogenase. Environ. Microbiol. 18, 191-204. doi: 10.1111/14622920.12960

Zhou, J. M., Wu, S. G., Qi, G. H., Fu, Y., Wang, W. W., Zhang, H. J., et al. (2020). Supplemental xylooligosaccharide modulates nutrient digestibility, intestinal morphology, and gut microbiota in laying hens. Anim. Nutr. 97, 874-881.

Conflict of Interest: The authors declare that the research was conducted in the absence of any commercial or financial relationships that could be construed as a potential conflict of interest.

Copyright (c) 2021 Zhou, Zhang, Wu, Qiu, Fu, Qi and Wang. This is an open-access article distributed under the terms of the Creative Commons Attribution License (CC BY). The use, distribution or reproduction in other forums is permitted, provided the original author(s) and the copyright owner(s) are credited and that the original publication in this journal is cited, in accordance with accepted academic practice. No use, distribution or reproduction is permitted which does not comply with these terms. 